Homology, Homotopy and Applications, vol.15(1), 2013, pp.151-189

\title{
PROFINITE G-SPECTRA
}

\section{GEREON QUICK}

\author{
(communicated by Daniel Dugger)
}

\begin{abstract}
We construct a stable model structure on profinite spectra with a continuous action of an arbitrary profinite group. The motivation is to provide a natural framework in a subsequent paper for a new and conceptually simplified construction of continuous homotopy fixed point spectra and of continuous homotopy fixed point spectral sequences for Lubin-Tate spectra under the action of the extended Morava stabilizer group.
\end{abstract}

\section{Introduction}

Let $G$ be a profinite group. A pointed profinite $G$-space is a simplicial object in the category of profinite sets with a continuous $G$-action together with a basepoint fixed under $G$. For example, the simplicial circle $S^{1}=\Delta^{1} / \partial \Delta^{1}$ is a pointed simplicial finite set. Hence when we consider $S^{1}$ with the trivial $G$-action it is an object of $\hat{\mathcal{S}}_{*}$. Pointed profinite $G$-spaces form a category which we denote by $\hat{\mathcal{S}}_{* G}$. A profinite $G$-spectrum $X$ is a sequence of pointed profinite $G$-spaces $X_{n}$ with maps $S^{1} \wedge X_{n} \rightarrow X_{n+1}$ in $\hat{\mathcal{S}}_{* G}$ for all $n$.

We show that the category $\operatorname{Sp}\left(\hat{\mathcal{S}}_{* G}\right)$ of profinite $G$-spectra is equipped with a natural stable model structure induced by a simplicial model structure in which the weak equivalences between fibrant objects are determined by the underlying maps of spectra. We discuss the simplicial structure of $\operatorname{Sp}\left(\hat{\mathcal{S}}_{* G}\right)$ in detail and construct homotopy limits of profinite $G$-spectra. Our main result, crucial for the applications in $[\mathbf{2 2}]$, is that, for a finitely generated profinite group $G$, every $G$-spectrum with finite homotopy groups has a concrete model in the category of profinite $G$-spectra. More precisely, let $X$ be a Bousfield-Friedlander spectrum with a compatible $G$-action on each space $X_{n}$ and assume that the homotopy groups of $X$ are all finite. Then there is a functorial $G$-equivariant map $\varphi^{s}: X \rightarrow F_{G}^{s} X$ of spectra from $X$ to a profinite $G$-spectrum $F_{G}^{s} X$ built of simplicial finite discrete $G$-sets such that $F_{G}^{s} X$ is fibrant in $\operatorname{Sp}\left(\hat{\mathcal{S}}_{* G}\right)$ and $\varphi^{s}$ is a stable equivalence of underlying spectra.

Continuous actions of a profinite group on spectra have been studied in a different setting for example by Davis in [5]. Davis considers discrete $G$-spectra whose

The author was supported by Research Fellowship QU 317/1 of the German Research Foundation (DFG).

Received March 13, 2011, revised September 27, 2012; published on April 28, 2013.

2000 Mathematics Subject Classification: 55P43, 55Q70, 55Q91.

Key words and phrases: profinite space, profinite spectrum, continuous group action, profinite homotopy type.

Article available at http://intlpress.com/HHA/v15/n1/a9 and doi:10.4310/HHA.2013.v15.n1.a9

Copyright (C) 2013, International Press. Permission to copy for private use granted. 
spaces are simplicial discrete $G$-sets. But for the purposes of homotopy fixed points and homotopy orbits under a profinite group action, the category of profinite $G$ spectra has the following important feature. The universal principal $G$-space $E G$ is an object in $\hat{\mathcal{S}}_{G}$ and the principal $G$-fibration $E G \rightarrow B G$ is a fibration in $\hat{\mathcal{S}}_{G}$. Using the fibrant replacement functor $R_{G}$ in $\operatorname{Sp}\left(\hat{\mathcal{S}}_{* G}\right)$, we can define the continuous homotopy fixed point spectrum of a profinite $G$-spectrum $X$ as a continuous mapping spectrum $X^{h G}=\operatorname{Map}_{G}\left(E G_{+}, R_{G} X\right)$ of $G$-equivariant and levelwise continuous maps in $\operatorname{Sp}\left(\hat{\mathcal{S}}_{* G}\right)$. The homotopy fixed point spectral sequence is then obtained just as for a finite group by filtering $E G$ by its finite subskeleta. Moreover, homotopy orbits can be defined as the quotient $X_{h G}=E G \times_{G} X$.

There are two main examples of a profinite group acting on a spectrum we have in mind. The first example is the action of the absolute Galois group on spectra arising in étale homotopy theory. In this case it is important to have a good notion of homotopy orbit spectra and homotopy orbit spectral sequences (see [18] and [20]). The second example is provided by the Morava stabilizer group and Lubin-Tate spectra. In $[\mathbf{2 2}]$ we use the present stable model structure on profinite $G$-spectra to give a new and conceptually simplified construction of continuous homotopy fixed points and homotopy fixed point spectral sequences for Lubin-Tate spectra.

Christensen and Isaksen [4] developed a model category structure on pro-spectra, which are different than our setting of profinite spectra. We give a comparison functor between pro-spectra and profinite spectra in Section 4.5.

\section{Acknowledgements}

I would like to thank Mike Hopkins for helpful discussions.

\section{Homotopy theory of profinite spaces}

\subsection{Profinite spaces}

We start with basic definitions and invariants for profinite spaces that will be necessary to construct a homotopy category for profinite spectra.

For a category $\mathcal{C}$ with small limits, the pro-category of $\mathcal{C}$, denoted pro- $\mathcal{C}$, has as objects all cofiltering diagrams $X: I \rightarrow \mathcal{C}$. Its sets of morphisms are defined as

$$
\operatorname{Hom}_{\text {pro- } \mathcal{C}}(X, Y):=\lim _{j \in J} \operatorname{colim}_{i \in I} \operatorname{Hom}_{\mathcal{C}}(X(i), Y(j)) \text {. }
$$

A constant pro-object is one indexed by the category with one object and one identity map. The functor sending an object $X$ of $\mathcal{C}$ to the constant pro-object with value $X$ makes $\mathcal{C}$ a full subcategory of pro- $\mathcal{C}$. The right adjoint of this embedding is the limit functor lim: pro- $\mathcal{C} \rightarrow \mathcal{C}$, which sends a pro-object $X$ to the limit in $\mathcal{C}$ of the diagram $X$.

Let $\mathcal{E}$ denote the category of sets and let $\mathcal{F}$ be the full subcategory of finite sets. Let $\hat{\mathcal{E}}$ be the category of compact Hausdorff and totally disconnected topological spaces. We may identify $\mathcal{F}$ with a full subcategory of $\hat{\mathcal{E}}$ in the obvious way. The limit functor $\lim$ : pro- $\mathcal{F} \rightarrow \hat{\mathcal{E}}$ is an equivalence of categories. Moreover the forgetful functor $\hat{\mathcal{E}} \rightarrow \mathcal{E}$ admits a left adjoint $(\hat{.)}: \mathcal{E} \rightarrow \hat{\mathcal{E}}$ which is called profinite completion. 
We denote by $\hat{\mathcal{S}}$ (resp., $\mathcal{S}$ ) the category of simplicial profinite sets (resp., simplicial sets). The objects of $\hat{\mathcal{S}}$ (resp., $\mathcal{S}$ ) will be called profinite spaces (resp., spaces). The category $\hat{\mathcal{S}}$ was studied for the first time by Morel in $[\mathbf{1 6}]$.

For a profinite space $X$, we define the set $\mathcal{R}(X)$ of simplicial open equivalence relations on $X$. An element $R$ of $\mathcal{R}(X)$ is a simplicial profinite subset of the product $X \times X$ such that, in each degree $n, R_{n}$ is an equivalence relation on $X_{n}$ and an open subset of $X_{n} \times X_{n}$. It is ordered by inclusion. For every element $R$ of $\mathcal{R}(X)$, the quotient $X / R$ is a simplicial finite set and the map $X \rightarrow X / R$ is a map of profinite spaces. The canonical map $X \rightarrow \lim _{R \in \mathcal{R}(X)} X / R$ is an isomorphism in $\hat{\mathcal{S}}$.

The profinite completion of sets induces a functor $(\hat{)}): \mathcal{S} \rightarrow \hat{\mathcal{S}}$, which is also called profinite completion. For a space $Z$, its profinite completion can be described as follows. Let $\mathcal{R}(Z)$ be the set of simplicial equivalence relations $R$ on $Z$ such that the quotient $Z / R$ is a simplicial finite set. The set $\mathcal{R}(Z)$ is ordered by inclusion. The profinite completion is defined as the limit of the $Z / R$ for all $R \in \mathcal{R}(Z)$, i.e., $\hat{Z}:=\lim _{R \in \mathcal{R}(Z)} Z / R$. Profinite completion of spaces is again left adjoint to the forgetful functor $|\cdot|: \hat{\mathcal{S}} \rightarrow \mathcal{S}$ which sends a profinite space to its underlying simplicial set.

Let $X$ be a profinite space and $\pi$ a topological abelian group. The continuous cohomology $H^{*}(X ; \pi)$ of $X$ with coefficients in $\pi$ is defined as the cohomology of the complex $C^{*}(X ; \pi)$ of continuous cochains of $X$ with values in $\pi$, i.e., $C^{n}(X ; \pi)$ denotes the set $\operatorname{Hom}_{\text {cont }}\left(X_{n}, \pi\right)$ of continuous maps $\alpha: X_{n} \rightarrow \pi$ and the differentials $\delta^{n}: C^{n}(X ; \pi) \rightarrow C^{n+1}(X ; \pi)$ are the morphisms associating to $\alpha$ the map $\sum_{i=0}^{n+1}(-1)^{i} \alpha \circ d_{i}$, where $d_{i}$ denotes the $i$ th face map $X_{n+1} \rightarrow X_{n}$. If $\pi$ is a finite abelian group and $Z$ a simplicial set, then the cohomology $H^{*}(Z ; \pi)$ of the space $Z$ and the continuous cohomology $H^{*}(\hat{Z} ; \pi)$ of the profinite completion $\hat{Z}$ are canonically isomorphic.

Convention 2.1. Above and in the rest of the paper we do not use a special notation for continuous cohomology. For a profinite space and a topological coefficient group, cohomology will always mean continuous cohomology.

If $G$ is an arbitrary profinite group, we may still define the first cohomology of $X$ with coefficients in $G$ following Morel's idea in [16], p. 355. The functor $(\hat{\mathcal{S}})^{\text {op }} \rightarrow \mathcal{E}$, $X \mapsto \operatorname{Hom}_{\hat{\mathcal{E}}}\left(X_{0}, G\right)$, is represented in $\hat{\mathcal{S}}$ by the profinite space $E G$, whose set of $n$ simplices is $E G_{n}=G^{n+1}$, the $(n+1)$-fold product of $G$. We define the 1-cocycles $Z^{1}(X ; G)$ to be the set of continuous maps $f: X_{1} \rightarrow G$ such that $f\left(d_{0} x\right) f\left(d_{2} x\right)=$ $f\left(d_{1} x\right)$ for every $x \in X_{1}$. The functor $(\hat{\mathcal{S}})^{\text {op }} \rightarrow \mathcal{E}, X \mapsto Z^{1}(X ; G)$ is represented by a profinite space $B G=E G / G$, whose set of $n$-simplices is $B G_{n}=G^{n}$, the $n$-fold product of $G$. Furthermore, there is a map

$$
\delta: \operatorname{Hom}_{\hat{\mathcal{S}}}(X, E G) \rightarrow Z^{1}(X ; G) \cong \operatorname{Hom}_{\hat{\mathcal{S}}}(X, B G)
$$

which sends $f: X_{0} \rightarrow G$ to the 1-cocycle $x \mapsto \delta f(x)=f\left(d_{0} x\right) f\left(d_{1} x\right)^{-1}$. We denote by $B^{1}(X ; G)$ the image of $\delta$ in $Z^{1}(X ; G)$ and we define the pointed set $H^{1}(X ; G)$ to be the quotient $Z^{1}(X ; G) / B^{1}(X ; G)$. Finally, if $X$ is a profinite space, we define $\pi_{0} X$ to be the coequalizer in $\mathcal{E}$ of the diagram $d_{0}, d_{1}: X_{1} \rightrightarrows X_{0}$.

The profinite fundamental group of $X$ is defined via covering spaces in the spirit of Grothendieck. There is a universal profinite covering space $(\tilde{X}, x)$ of $X$ at a vertex 
$x \in X_{0}$. Then $\pi_{1}(X, x)$ is defined to be the group of automorphisms of $(\tilde{X}, x)$ over $(X, x)$. As the limit of the finite automorphism groups of the finite Galois coverings of $(X, x)$ the group $\pi_{1}(X, x)$ is naturally a profinite group. For details we refer the reader to $[\mathbf{1 9}]$.

Definition 2.2. A morphism $f: X \rightarrow Y$ in $\hat{\mathcal{S}}$ is called

- a weak equivalence if the induced map $f_{*}: \pi_{0}(X) \rightarrow \pi_{0}(Y)$ is an isomorphism of profinite sets, $f_{*}: \pi_{1}(X, x) \rightarrow \pi_{1}(Y, f(x))$ is an isomorphism of profinite groups for every vertex $x \in X_{0}$, and $f^{*}: H^{q}(Y ; \mathcal{M}) \rightarrow H^{q}\left(X ; f^{*} \mathcal{M}\right)$ is an isomorphism for every local coefficient system $\mathcal{M}$ of finite abelian groups on $Y$ for every $q \geqslant 0$

- a cofibration if $f$ is a levelwise monomorphism;

- a fibration if it has the right lifting property with respect to every cofibration that is also a weak equivalence.

The category $\hat{\mathcal{S}}$ has a simplicial structure (see also [7], $\S 1.2$ ). Let $X$ and $Y$ be profinite spaces. The mapping space $\operatorname{map}_{\hat{\mathcal{S}}}(X, Y)$ is defined as the simplicial set whose set of $n$-simplices is given as the set of maps

$$
\operatorname{map}_{\hat{\mathcal{S}}}(X, Y)_{n}=\operatorname{Hom}_{\hat{\mathcal{S}}}(X \times \Delta[n], Y)
$$

and whose simplicial structure is induced by the cosimplicial structure of the standard simplex $[n] \mapsto \Delta[n]$. This defines a functor

$$
\operatorname{map}_{\hat{\mathcal{S}}}(-,-): \hat{\mathcal{S}}^{\mathrm{op}} \times \hat{\mathcal{S}} \rightarrow \mathcal{S} .
$$

The tensor and cotensor structure on the category $\hat{\mathcal{S}}$ is defined as follows. Let $K$ be a finite simplicial set, i.e., a simplicial set with only finitely many nondegenerate simplices. This implies, in particular, that $K$ is a simplicial finite set. Let $X$ be a profinite space. The tensor object $X \otimes K \in \hat{\mathcal{S}}$ is defined as the levelwise defined product of simplicial profinite sets $X \times K$. For the cotensor object, we recall that any profinite space $X$ is canonically isomorphic to a $\operatorname{limit} \lim _{\beta} X_{\beta}$ of simplicial finite sets. This implies that the set of maps $\operatorname{Hom}_{\hat{\mathcal{S}}}(K, X)$ inherits a natural structure as a profinite set given by the limit of finite sets

$$
\lim _{\beta} \operatorname{Hom}_{\hat{\mathcal{S}}}\left(K, X_{\beta}\right) .
$$

The cotensor or function object in $\hat{\mathcal{S}}$ is defined as the profinite space $\operatorname{hom}_{\hat{\mathcal{S}}}(K, X) \in \hat{\mathcal{S}}$ whose set of $n$-simplices is given by the profinite set of maps

$$
\operatorname{hom}_{\hat{\mathcal{S}}}(K, X)_{n}=\operatorname{Hom}_{\hat{\mathcal{S}}}(K \times \Delta[n], X) .
$$

If $K$ is an arbitrary simplicial set, it is isomorphic to the filtered colimit over its finite simplicial subsets $K_{\alpha}$. For a profinite space $X$, we define the tensor object $X \otimes K$ to be the colimit in $\hat{\mathcal{S}}$ of the profinite spaces $X \otimes K_{\alpha}$ (see also [7], §1.2), i.e.,

$$
X \otimes K:=\operatorname{colim}_{\alpha} X \times K_{\alpha} \text { in } \hat{\mathcal{S}} \text {. }
$$

The function object is defined to be the limit in $\hat{\mathcal{S}}$ of the profinite spaces 
$\operatorname{hom}_{\hat{\mathcal{S}}}\left(K_{\alpha}, X\right)$, i.e.,

$$
\operatorname{hom}_{\hat{\mathcal{S}}}(K, X):=\lim _{\alpha} \operatorname{hom}_{\hat{\mathcal{S}}}\left(K_{\alpha}, X\right) \text { in } \hat{\mathcal{S}} .
$$

Let $X$ and $Y$ be profinite spaces and let $K$ be a simplicial set. Then mapping space, tensor and function objects are connected by the natural bijections

$$
\operatorname{map}_{\hat{\mathcal{S}}}(X \otimes K, Y) \cong \operatorname{map}_{\mathcal{S}}\left(K, \operatorname{map}_{\hat{\mathcal{S}}}(X, Y)\right)
$$

and

$$
\operatorname{map}_{\hat{\mathcal{S}}}\left(Y, \operatorname{hom}_{\hat{\mathcal{S}}}(K, X)\right) \cong \operatorname{map}_{\mathcal{S}}\left(K, \operatorname{map}_{\hat{\mathcal{S}}}(X, Y)\right)
$$

where $\operatorname{map}_{\mathcal{S}}(-,-)$ denotes the mapping space functor on $\mathcal{S}$. This defines the structure of a simplicial category on $\hat{\mathcal{S}}$ in the sense of [23], II $\S \S 1+2$.

The following theorem was proven in [20], Theorem 2.3.

Theorem 2.3. The classes of weak equivalences, cofibrations and fibrations, as defined above, provide $\hat{\mathcal{S}}$ with the structure of a fibrantly generated left proper simplicial model category. We denote the homotopy category by $\hat{\mathcal{H}}$.

We consider the category $\mathcal{S}$ of simplicial sets with the usual model structure of [23]. We denote its homotopy category by $\mathcal{H}$. Then the next result was shown in [19].

\section{Proposition 2.4.}

(a) The levelwise completion functor $(\hat{\cdot}): \mathcal{S} \rightarrow \hat{\mathcal{S}}$ preserves weak equivalences and cofibrations.

(b) The forgetful functor $|\cdot|: \hat{\mathcal{S}} \rightarrow \mathcal{S}$ preserves fibrations and weak equivalences between fibrant objects.

(c) The induced completion functor $\hat{(\cdot)}: \mathcal{H} \rightarrow \hat{\mathcal{H}}$ and the right derived functor $R|\cdot|: \hat{\mathcal{H}} \rightarrow \mathcal{H}$ form a pair of adjoint functors.

\subsection{Pointed profinite spaces}

Let $\hat{\mathcal{S}}_{*}$ be the category of pointed profinite spaces, i.e., profinite spaces $X$ with a chosen map from the one-point space to $X$. Profinite completion of spaces induces in the obvious way a profinite completion functor $\hat{(\cdot)}: \mathcal{S}_{*} \rightarrow \hat{\mathcal{S}}_{*}$, where $\mathcal{S}_{*}$ denotes the category of pointed spaces.

For pointed profinite spaces $X$ and $Y$, let $X \vee Y$ be the wedge of $X$ and $Y$ over the base point. The smash product $X \wedge Y$ is defined to be the quotient in $\hat{\mathcal{S}}_{*}$

$$
X \wedge Y:=(X \times Y) /(X \vee Y) \text {. }
$$

The category $\hat{\mathcal{S}}_{*}$ also has a simplicial structure (cf. again [7], $\left.\S 1.2\right)$. Let $X$ and $Y$ be

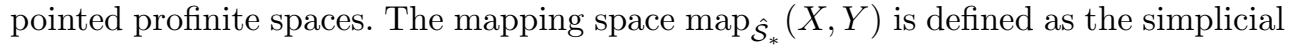
set whose set of $n$-simplices is given as the set of maps

$$
\operatorname{map}_{\hat{\mathcal{S}}_{*}}(X, Y)_{n}=\operatorname{Hom}_{\hat{\mathcal{S}}_{*}}\left(X \wedge \Delta[n]_{+}, Y\right)
$$

where $\Delta[n]_{+}$denotes the standard simplicial set with an additional disjoint base point. This defines a functor

$$
\operatorname{map}_{\hat{\mathcal{S}}_{*}}(-,-): \hat{\mathcal{S}}_{*}^{\mathrm{op}} \times \hat{\mathcal{S}}_{*} \rightarrow \mathcal{S} .
$$

The tensor and cotensor structure are defined as follows. Let $K$ be a finite simplicial set, and $X$ a pointed profinite space. The tensor object $X \otimes K \in \hat{\mathcal{S}}_{*}$ is defined as the 
smash product $X \wedge K_{+}$. The function object in $\hat{\mathcal{S}}_{*}$ is defined as the pointed profinite space $\operatorname{hom}_{\hat{\mathcal{S}}_{*}}(K, X) \in \hat{\mathcal{S}}_{*}$, pointed by the constant map to the basepoint of $X$, whose set of $n$-simplices is given by the profinite set of maps

$$
\operatorname{hom}_{\hat{\mathcal{S}}_{*}}(K, X)_{n}=\operatorname{Hom}_{\hat{\mathcal{S}}_{*}}\left(K_{+} \wedge \Delta[n]_{+}, X\right) .
$$

If $K$ is an arbitrary simplicial set and $X$ a pointed profinite space, we define the tensor object $X \otimes K$ to be the colimit in $\hat{\mathcal{S}}_{*}$ of the pointed profinite spaces $X \wedge K_{\alpha+}$ where $K_{\alpha}$ runs through the finite simplicial subsets of $K$ (see also [7], §1.2).

The function object $\operatorname{hom}_{\hat{\mathcal{S}}_{*}}(K, X)$ is defined to be the limit in $\hat{\mathcal{S}}_{*}$ of the pointed profinite spaces $\operatorname{hom}_{\hat{\mathcal{S}}_{*}}\left(K_{\alpha}, X\right)$.

Let $X$ and $Y$ be pointed profinite spaces and let $K$ be a simplicial set. Then mapping space, tensor and function objects are connected by the natural bijections

$$
\operatorname{map}_{\hat{\mathcal{S}}_{*}}(X \otimes K, Y) \cong \operatorname{map}_{\mathcal{S}}\left(K, \operatorname{map}_{\hat{\mathcal{S}}_{*}}(X, Y)\right)
$$

and

$$
\operatorname{map}_{\hat{\mathcal{S}}_{*}}\left(Y, \operatorname{hom}_{\hat{\mathcal{S}}_{*}}(K, X)\right) \cong \operatorname{map}_{\mathcal{S}}\left(K, \operatorname{map}_{\hat{\mathcal{S}}_{*}}(X, Y)\right) .
$$

This defines the structure of a simplicial category on $\hat{\mathcal{S}}_{*}$.

If the finite simplicial set $K$ is already equipped with a base point and $X$ is a pointed profinite space, we also denote by $\operatorname{hom}_{\hat{\mathcal{S}}_{*}}(K, X) \in \hat{\mathcal{S}}_{*}$ the pointed profinite space whose set of $n$-simplices is given by the profinite set of maps

$$
\operatorname{hom}_{\hat{\mathcal{S}}_{*}}(K, X)_{n}=\operatorname{Hom}_{\hat{\mathcal{S}}_{*}}\left(K \wedge \Delta[n]_{+}, X\right) .
$$

Moreover, if $X$ is a pointed profinite space and $K$ is an arbitrary pointed simplicial set, we define the pointed profinite space $\operatorname{hom}_{\hat{\mathcal{S}}_{*}}(K, X) \in \hat{\mathcal{S}}_{*}$ to be the limit in $\hat{\mathcal{S}}_{*}$

$$
\operatorname{hom}_{\hat{\mathcal{S}}_{*}}(K, X)=\lim _{\alpha} \operatorname{hom}_{\hat{\mathcal{S}}_{*}}\left(K_{\alpha}, X\right)
$$

where $K_{\alpha}$ runs through the pointed finite simplicial subsets of $K$ such that $K$ is isomorphic to $\operatorname{colim}_{\alpha} K_{\alpha}$ as a pointed simplicial set.

If $K$ and $L$ are pointed simplicial sets and $X$ a pointed profinite space, we also have the following natural isomorphism

$$
\operatorname{hom}_{\hat{\mathcal{S}}_{*}}\left(K, \operatorname{hom}_{\hat{\mathcal{S}}_{*}}(L, X)\right) \cong \operatorname{hom}_{\hat{\mathcal{S}}_{*}}(K \wedge L, X) \cong \operatorname{hom}_{\hat{\mathcal{S}}_{*}}\left(L, \operatorname{hom}_{\hat{\mathcal{S}}_{*}}(K, X)\right) .
$$

Example 2.5. As an example of function objects, let $S^{1}$ be the simplicial circle, i.e., the quotient $S^{1}=\Delta[1] / \partial \Delta[1]$ of the standard simplex $\Delta[1]$ by its boundary. The pointed simplicial set $S^{1}$ is finite in each degree, i.e., it is a simplicial finite set and hence also an object in $\hat{\mathcal{S}}_{*}$. Taking the smash product with $S^{1}$ defines a functor $\hat{\mathcal{S}}_{*} \rightarrow \hat{\mathcal{S}}_{*}, X \mapsto S^{1} \wedge X$. It is left adjoint to the functor $\hat{\mathcal{S}}_{*} \rightarrow \hat{\mathcal{S}}_{*}$ defined by sending a pointed profinite space $X$ to the function object $\operatorname{hom}_{\hat{\mathcal{S}}_{*}}\left(S^{1}, X\right)$ in $\hat{\mathcal{S}}_{*}$. We denote $\operatorname{hom}_{\hat{\mathcal{S}}_{*}}\left(S^{1}, X\right)$ also by $\Omega X$. Hence for pointed profinite spaces $X$ and $Y$ there is a natural isomorphism

$$
\operatorname{map}_{\hat{\mathcal{S}}_{*}}\left(S^{1} \wedge X, Y\right) \cong \operatorname{map}_{\hat{\mathcal{S}}_{*}}(X, \Omega Y) .
$$

As an under-category of $\hat{\mathcal{S}}, \hat{\mathcal{S}}_{*}$ inherits a model structure. We call a map in $\hat{\mathcal{S}}_{*}$ a weak equivalence (cofibration, fibration) if its underlying map in $\hat{\mathcal{S}}$ is a weak equivalence (cofibration, fibration). It follows from the general theory of model categories, 
as explained for example in [13], Proposition 1.1.8 and the dual of Lemma 2.1.21, that Theorem 2.3 implies the following result.

Theorem 2.6. The classes of weak equivalences, cofibrations and fibrations provide $\hat{\mathcal{S}}_{*}$ with the structure of a fibrantly generated left proper simplicial model category. We denote the homotopy category by $\hat{\mathcal{H}}_{*}$.

The Quillen adjunction of Proposition 2.4 can be rephrased in terms of mapping spaces. Let $K$ be a pointed simplicial set and $X$ be a fibrant pointed profinite space. Then there is a natural isomorphism of fibrant simplicial sets

$$
\operatorname{map}_{\hat{\mathcal{S}}_{*}}(\hat{K}, X) \cong \operatorname{map}_{\mathcal{S}_{*}}(K,|X|) .
$$

Moreover, if $K=S^{1}$ is the simplicial circle, there is an isomorphism in $\mathcal{S}_{*}$

$$
|\Omega X|=\left|\operatorname{hom}_{\hat{\mathcal{S}}_{*}}\left(S^{1}, X\right)\right| \cong \operatorname{map}_{\hat{\mathcal{S}}_{*}}\left(S^{1}, X\right) \cong \operatorname{map}_{\mathcal{S}_{*}}\left(S^{1},|X|\right)=: \Omega|X| .
$$

Remark 2.7. Let $X$ be a pointed profinite space. The maps $d_{0}, d_{1}: \Delta[0] \rightrightarrows \Delta[1]$ and $s^{0}: \Delta[1] \rightarrow \Delta[0]$ induce maps $X \vee X \rightarrow X \wedge \Delta[1]_{+}$and $X \wedge \Delta[1]_{+} \rightarrow X$ and make $X \wedge \Delta[1]_{+}$into a cylinder object for $X$ in $\hat{\mathcal{S}}_{*}$. For the maps $X \rightarrow X \wedge \Delta[1]_{+}$induced by $d_{0}$ and $d_{1}$ are cofibrations and weak equivalences and the induced map $X \wedge \Delta[1]_{+} \rightarrow X$ is a weak equivalence in $\hat{\mathcal{S}}_{*}$. Hence if $Y$ is a fibrant pointed profinite space, there is a natural bijection

$$
\pi_{0} \operatorname{map}_{\hat{\mathcal{S}}_{*}}(X, Y) \cong \operatorname{Hom}_{\hat{\mathcal{H}}_{*}}(X, Y)
$$

Moreover, let $K$ be a pointed simplicial set, $X, Y$ pointed profinite spaces, and $Y$ fibrant in $\hat{\mathcal{S}}_{*}$. If we denote the homotopy category of pointed simplicial sets by $\mathcal{H}_{*}$, then there are natural bijections

$$
\begin{gathered}
\operatorname{Hom}_{\hat{\mathcal{H}}_{*}}(K \otimes X, Y) \cong \operatorname{Hom}_{\hat{\mathcal{H}}_{*}}\left(X, \operatorname{hom}_{\hat{\mathcal{S}}_{*}}(K, Y)\right), \\
\operatorname{Hom}_{\hat{\mathcal{H}}_{*}}(K \otimes X, Y) \cong \operatorname{Hom}_{\mathcal{H}_{*}}\left(K, \operatorname{map}_{\hat{\mathcal{S}}_{*}}(X, Y)\right), \text { and } \\
\operatorname{Hom}_{\hat{\mathcal{H}}_{*}}(\hat{K}, Y) \cong \operatorname{Hom}_{\mathcal{H}_{*}}(K,|Y|) .
\end{gathered}
$$

\subsection{An explicit fibrant replacement functor}

As indicated by Morel for pro-p-completion of spaces in [16], $\{2.1$, p. 367, there is an explicit construction for the fibrant replacement in $\hat{\mathcal{S}}$. This construction is based on the work of Quillen [24] and Dwyer-Kan [9]. We refer the reader to [21] for more details.

First, let $X$ be a reduced simplicial finite set. We denote by $\Gamma X$ the free loop group construction of $X$. When we apply the profinite completion of groups levelwise to the simplicial group $\Gamma X$, we obtain a simplicial profinite group denoted by $\hat{\Gamma} X$. Its profinite classifying space $\bar{W} \hat{\Gamma} X$ is a fibrant object in $\hat{\mathcal{S}}$. It is equipped with a canonical map $\eta: X \rightarrow \bar{W} \hat{\Gamma} X$ and one can show that this is a weak equivalence of profinite spaces. The crucial point is that free groups are good in the sense of Serre which we will discuss below.

Second, let $X$ be an arbitrary simplicial finite set. We can generalize the previous argument by applying to $X$ the free loop groupoid construction $\Gamma X$ (see [9] or [11], $\mathrm{V} \S 7$ ). It is defined in degree $n$ to be the free groupoid on generators $x: x_{1} \rightarrow x_{0}$ with $x \in X_{n+1}$, subject to the relations $s_{0} x_{0}=1_{x_{0}}, x_{0} \in X_{n}$. The objects of this groupoid 
are just the vertices of $\mathrm{X}$. One can define a functor $\Gamma X_{n} \rightarrow \Gamma X_{m}$ for each ordinal number morphism $[m] \rightarrow[n]$. Then we apply the degreewise profinite completion functor to groupoids. We call a groupoid $H$ finite if the set of objects of $H$ is finite and the group of automorphisms of any object is finite as well. The profinite completion of a groupoid is then the limit as a groupoid of the filtered system of its quotient groupoids which are finite groupoids in this sense (see also [16], p. 367). We obtain a simplicial profinite groupoid $\hat{\Gamma} X$. We apply again the classifying space functor $\bar{W}$ for simplicial groupoids of $[\mathbf{1 1}], \mathrm{V}$, to get the fibrant profinite space $\bar{W} \hat{\Gamma} X$ and a canonical map $\eta: X \rightarrow \bar{W} \hat{\Gamma} X$ in $\hat{\mathcal{S}}$.

Finally, let $X$ be an arbitrary profinite space. It is isomorphic in $\hat{\mathcal{S}}$ to the limit $\lim _{Q} X / Q$ where $Q$ runs through the simplicial open equivalence relations on $X$, i.e., those simplicial relations such that $Q_{n}$ is an open subset of $X_{n} \times X_{n}$ for each $n$. We define its free simplicial profinite groupoid by

$$
\hat{G \Gamma} X:=\lim _{Q} \hat{\Gamma}(X / Q) .
$$

Then we apply $\bar{W}$ to get a fibrant profinite space $R X$ equipped with a canonical map

$$
\eta: X \rightarrow R X:=\bar{W} \hat{\Gamma} X=\lim _{Q} \bar{W} \hat{\Gamma}(X / Q) .
$$

As before one can show that $\eta$ is a weak equivalence. Hence $\eta$ gives a fibrant replacement functor in $\hat{\mathcal{S}}$. In particular, we can write $R X$ as a limit

$$
R X=\lim _{Q} \lim _{U_{Q}} \bar{W}\left(\Gamma(X / Q) / U_{Q}\right)
$$

where $U_{Q}$ runs through the normal subgroupoids of $\Gamma(X / Q)$ such that the quotient $\Gamma(X / Q) / U_{Q}$ is a simplicial finite groupoid. Hence we can decompose a fibrant profinite space into a limit of simplicial finite sets which are fibrant in $\hat{\mathcal{S}}$. Moreover, after taking Postnikov sections we can write $R X$ as a limit

$$
R X=\lim _{Q, n} \lim _{U_{Q}} \operatorname{cosk}_{n} \bar{W}\left(\Gamma(X / Q) / U_{Q}\right)
$$

of simplicial finite sets which are also finite spaces, i.e., simplicial sets whose homotopy groups are all finite and only a finite number of them are nontrivial.

Finally, let $X$ be a pointed profinite space with basepoint given by a map $p: * \rightarrow X$ from the discrete simplicial set $*$ with a single vertex. The decomposition of $X$ as a limit of simplicial finite sets $X \cong \lim _{Q} X / Q$ is then also a decomposition as a limit of pointed simplicial finite sets, since $p$ induces compatible maps $* \rightarrow X / Q$ which provide basepoints for each $X / Q$. Hence the map

$$
\eta: X \rightarrow R X
$$

provides in fact a fibrant replacement in the model structure of pointed profinite spaces such that $R X$ is a limit of pointed simplicial finite sets which are fibrant in $\hat{\mathcal{S}}_{*}$.

\subsection{Homotopy groups of profinite spaces}

Let $X$ be a pointed profinite space. The profinite fundamental group has been defined above. For $n \geqslant 2$, we define the $n$th homotopy group of $X$ as follows. 
Definition 2.8. Let $X$ be a pointed profinite space and let $R X$ be a fibrant replacement of $X$ in the above model structure on $\hat{\mathcal{S}}_{*}$. For $n \geqslant 2$, we define the $n$th profinite homotopy group of $X$ to be the group

$$
\pi_{n}(X):=\pi_{0}\left(\Omega^{n}(R X)\right) .
$$

As shown in [19], this is consistent with the definition of $\pi_{1}$ as well. For $n \geqslant 2$, the higher homotopy groups $\pi_{n} X$ are abelian groups, since $\Omega^{n} X$ is a group object in $\hat{\mathcal{H}}_{*}$.

Lemma 2.9. Let $X$ be a fibrant pointed profinite space. The profinite homotopy groups of $X$ defined in Definition 2.8 are naturally isomorphic to the usual homotopy groups of the underlying fibrant simplicial set $|X|$.

Proof. By Proposition 2.4, the forgetful functor $|\cdot|: \hat{\mathcal{S}}_{*} \rightarrow \mathcal{S}_{*}$ preserves fibrant objects and weak equivalences between fibrant objects. Since $X$ is a fibrant pointed profinite space, for every $n \geqslant 0$ we have an isomorphism of groups (respectively sets for $n=0$ )

$$
\pi_{n}(X)=\pi_{0}\left(\Omega^{n}(X)\right) \cong \pi_{0}\left(\Omega^{n}(|X|)\right) \cong \pi_{n}(|X|)
$$

where $\pi_{n}(|X|)$ denotes the $n$th homotopy group of the simplicial set $|X|$.

Remark 2.10. The assumption in the previous lemma that $X$ is fibrant in $\hat{\mathcal{S}}_{*}$ is of course crucial. If we start with an arbitrary pointed profinite space $Y$, then the homotopy group $\pi_{n}(|Y|)$ of its underlying space $|Y|$ is in general not isomorphic to the homotopy group $\pi_{n}(Y)$ of Definition 2.8. Since the notion of weak equivalences in $\hat{\mathcal{S}}_{*}$ is different from the one in $\mathcal{S}_{*}$, the fibrant replacement in $\hat{\mathcal{S}}_{*}$ does not preserve the homotopy type of $|Y|$ in $\mathcal{H}_{*}$.

Furthermore, the homotopy groups of Definition 2.8 carry a natural profinite structure. To simplify notations, let $X$ be fibrant in $\hat{\mathcal{S}}_{*}$. Using the explicit fibrant replacement functor defined in the previous section, we can assume that $X$ is given as the $\operatorname{limit}_{\lim _{\beta}} X_{\beta}$ of pointed simplicial finite sets which are fibrant in $\hat{\mathcal{S}}_{*}$. Since the underlying space of a fibrant object in $\hat{\mathcal{S}}_{*}$ is a fibrant simplicial set, we see that the spaces underlying $X$ and each underlying space of $X_{\beta}$ are fibrant as simplicial sets. Furthermore, since $\hat{\mathcal{S}}_{*}$ is a simplicial model category, $\operatorname{hom}_{\hat{\mathcal{S}}_{*}}\left(S^{1}, X_{\beta}\right)$ is a simplicial finite set which is fibrant in $\hat{\mathcal{S}}_{*}$. Hence

$$
\operatorname{hom}_{\hat{\mathcal{S}}_{*}}\left(S^{1}, X\right)=\operatorname{hom}_{\hat{\mathcal{S}}_{*}}\left(S^{1}, \lim _{\beta} X_{\beta}\right)=\lim _{\beta} \operatorname{hom}_{\hat{\mathcal{S}}_{*}}\left(S^{1}, X_{\beta}\right)
$$

is a cofiltered limit of fibrant simplicial finite sets. The fact that $\pi_{n}(X)$ is a profinite group is now implied by the following proposition due to Dehon and Lannes [6], Proposition 1.1.3.

Proposition 2.11. Let $\left\{X_{\beta}\right\}_{\beta}$ be a cofiltered diagram of fibrant simplicial finite sets. Then the canonical map

$$
\pi_{0}\left(\lim _{\beta} X_{\beta}\right) \rightarrow \lim _{\beta}\left(\pi_{0} X_{\beta}\right)
$$

is a bijection. 


\subsection{Homotopy limits of pointed profinite spaces}

Homotopy limits of diagrams of profinite spaces can be defined using the construction of Bousfield and Kan in [3], XI. Let $I$ be a small category and $B I$ be the nerve of $I$. Recall that this is the simplicial set whose $n$-simplices are sequences

$$
u=\left(i_{0} \stackrel{\alpha_{1}}{\leftarrow} \cdots \stackrel{\alpha_{n}}{\leftarrow} i_{n}\right)
$$

of morphisms in $I$. Moreover, for an object $i$ in $I$, one can form the over-category $I / i$ whose objects are maps $i_{0} \rightarrow i$. The nerve $B(I / i)$ of this over-category is the simplicial set whose $n$-simplices are sequences

$$
i \stackrel{\alpha}{\leftarrow} i_{0} \stackrel{\alpha_{1}}{\leftarrow} \ldots \stackrel{\alpha_{n}}{\leftarrow} i_{n}
$$

of morphisms in $I$.

Let $X(-): I \rightarrow \hat{\mathcal{S}}_{*}$ be a functor from $I$ to pointed profinite spaces. The existence of cotensor objects in $\hat{\mathcal{S}}_{*}$ allows to define the pointed profinite space

$$
\operatorname{hom}_{\hat{\mathcal{S}}_{*}}(B(I /-), X(-)) \in \hat{\mathcal{S}}_{*}
$$

as the equalizer in $\hat{\mathcal{S}}_{*}$ of the diagram of pointed profinite spaces

$$
\prod_{i \in I} \operatorname{hom}_{\hat{\mathcal{S}}_{*}}(B(I / i), X(i)) \rightrightarrows \prod_{\alpha: i \rightarrow i^{\prime} \in I} \operatorname{hom}_{\hat{\mathcal{S}}_{*}}\left(B(I / i), X\left(i^{\prime}\right)\right)
$$

where the two maps are induced by the maps in $\hat{\mathcal{S}}_{*}$

$$
\operatorname{hom}_{\hat{\mathcal{S}}_{*}}(B(I / i), X(i)) \stackrel{X(\alpha)}{\longrightarrow} \operatorname{hom}_{\hat{\mathcal{S}}_{*}}\left(B(I / i), X\left(i^{\prime}\right)\right)
$$

and

$$
\operatorname{hom}_{\hat{\mathcal{S}}_{*}}\left(B\left(I / i^{\prime}\right), X\left(i^{\prime}\right)\right) \stackrel{B(I / \alpha)}{\longrightarrow} \operatorname{hom}_{\hat{\mathcal{S}}_{*}}\left(B(I / i), X\left(i^{\prime}\right)\right) .
$$

In analogy to [3], XI $\S 3$, we make the following definition.

Definition 2.12. Let $I$ be small category and $X(-): I \rightarrow \hat{\mathcal{S}}_{*}$ be a functor. The homotopy limit of $X(-)$ is defined to be the pointed profinite space

$$
\operatorname{holim}_{i \in I} X(i):=\operatorname{hom}_{\hat{\mathcal{S}}_{*}}(B(I /-), X(-)) .
$$

Lemma 2.13. Let $X(-)$ be a functor from a small category $I$ to pointed profinite spaces. If $X(i)$ is fibrant for every $i \in I$, then $\operatorname{holim}_{i \in I} X(i)$ is fibrant in $\hat{\mathcal{S}}_{*}$. Let $f(-): X(-) \rightarrow Y(-)$ be a natural transformation of functors from the small category $I$ to the full subcategory of fibrant pointed profinite spaces. Let

$$
f:=\operatorname{holim}_{i \in I} f(i): \operatorname{holim}_{i \in I} X(i) \rightarrow \operatorname{holim}_{i \in I} Y(i)
$$

be the induced map on homotopy limits. If $f(i): X(i) \rightarrow Y(i)$ is a weak equivalence in $\hat{\mathcal{S}}_{*}$ for every $i \in I$, then $f$ is a weak equivalence in $\hat{\mathcal{S}}_{*}$.

Proof. This is proved in [12], Theorem 19.4.1 and Theorem 19.4.2. See also Remark 19.1.6 and Proposition 16.6.6 in [12] that show that the definition of homotopy limits in $[\mathbf{1 2}], \S 19$, coincides with the construction in Definition 2.12. 
Lemma 2.14. Let $X(-): I \rightarrow \hat{\mathcal{S}}_{*}$ be a small cofiltering diagram such that each $X(i)$ is connected and fibrant in $\hat{\mathcal{S}}_{*}$. Then there is a natural isomorphism of homotopy groups of underlying simplicial sets for every $q \geqslant 0$

$$
\pi_{q}\left(\left|\operatorname{holim}_{i \in I} X(i)\right|\right) \cong \lim _{i \in I} \pi_{q}(|X(i)|) .
$$

Proof. Since the right Quillen functor $|-|: \hat{\mathcal{S}}_{*} \rightarrow \mathcal{S}_{*}$ of forgetting the profinite structure commutes with limits and cotensor objects, it also commutes with forming the homotopy limit of the diagram $X(-): I \rightarrow \hat{\mathcal{S}}_{*}$, i.e., there is an isomorphism in $\mathcal{S}_{*}$

$$
\left|\operatorname{holim}_{i \in I} X(i)\right| \cong \operatorname{holim}_{i \in I}|X(i)| \text {. }
$$

By [3] XI $\S 7.1$, since each $|X(i)|$ is a fibrant pointed simplicial set, there is a spectral sequence involving derived limits

$$
E_{2}^{s, t}= \begin{cases}\lim _{I}^{s} \pi_{t}(|X(i)|) & \text { for } 0 \leqslant s \leqslant t \\ 0 & \text { else }\end{cases}
$$

converging to $\pi_{s+t} \operatorname{holim}_{i}|X(i)|$. As we pointed out in Lemma 2.9, the homotopy groups $\pi_{t}(|Y|)$ of the pointed simplicial set $|Y|$ underlying a fibrant pointed profinite space $Y$ satisfy

$$
\pi_{t}(|Y|) \cong \pi_{t}(Y)
$$

where the right hand group is the profinite homotopy group of the fibrant pointed profinite space $Y$ of Definition 2.8. This implies that the groups $\pi_{t}|X(i)|$ in (2) are profinite groups for all $t$ and all $i$. Since the functor sending a small cofiltering diagram to its inverse limit is exact on the category of profinite groups ([25] Proposition 2.2.4), spectral sequence (2) degenerates to a single row and implies the desired isomorphism.

\subsection{Comparison with the work of Artin-Mazur, Morel and Sullivan}

Let $\mathcal{H}_{\text {fin }}$ denote the full subcategory of $\mathcal{H}$ of finite spaces, i.e., of simplicial finite sets whose homotopy groups are all finite and only a finite number of them are nontrivial. The morphisms are homotopy classes of maps. Let $\mathcal{H}_{0}$ denote the full subcategory of connected spaces. Artin and Mazur showed in [1] that, for every space $X \in \mathcal{H}_{0}$, the functor

$$
\mathcal{H}_{\text {fin }} \rightarrow \mathcal{E}, F \mapsto[X, F],
$$

is pro-representable in $\mathcal{H}_{\text {fin }}$. The representing pro-object $\hat{X}^{\mathrm{AM}} \in$ pro $-\mathcal{H}_{\text {fin }}$ is called the (Artin-Mazur) profinite completion of $X$. Then Sullivan showed in [27] that the underlying diagram in $\mathcal{H}_{\text {fin }}$ of $\hat{X}^{\mathrm{AM}}$ has a limit $\hat{X}^{\mathrm{Su}}$ in $\mathcal{H}$, which is called the profinite completion of Sullivan (see also [16]).

The previously constructed fibrant replacement functor in $\hat{\mathcal{S}}$ for the model structure of Theorem 2.3 provides a rigid model of the Artin-Mazur and Sullivan profinite completion and extends it to nonconnected spaces. This rigidification is obtained as follows.

Given a profinite space $Z$, we have seen above that we can decompose it into an inverse system of fibrant profinite spaces with finite homotopy groups. Together with taking finite Postnikov sections, this yields a decomposition of $Z$ into an inverse 
system of finite spaces in $\mathcal{S}$. By applying this to the completion $\hat{X}$ of a space $X$, we get a functor

$$
T: \mathcal{S} \rightarrow \hat{\mathcal{S}} \rightarrow \text { pro }-\mathcal{S}_{\text {fin }}
$$

where $\mathcal{S}_{\text {fin }}$ is the full subcategory of $\mathcal{S}$ of finite spaces. Now we can consider this functor on the homotopy level

$$
T: \mathcal{H} \rightarrow \hat{\mathcal{H}} \rightarrow \text { pro }-\mathcal{H}_{\text {fin }} .
$$

It follows immediately from the previous results on profinite spaces and [1], Theorem 4.3, that $\left.T\right|_{\mathcal{H}_{0}}$ is isomorphic to the Artin-Mazur completion functor. Moreover, this implies that the fibrant replacement of $\hat{X}$ in $\hat{\mathcal{S}}$ is a rigid model for the Sullivan completion of $X$, i.e., that $|R \hat{X}|$ is canonically isomorphic to $\hat{X}^{\text {Su }}$ in $\mathcal{H}$. Hence $|R \hat{X}|$ provides a rigid model for profinite completion.

In [16] Morel proved that there is a model structure on $\hat{\mathcal{S}}$ for each prime number $p$ in which the weak equivalences are the maps that induce isomorphisms on continuous $\mathbb{Z} / p$-cohomology. The generating fibrations are given by the canonical maps $L(\mathbb{Z} / p, n) \rightarrow K(\mathbb{Z} / p, n+1), K(\mathbb{Z} / p, n) \rightarrow *$, and trivial fibrations are given by the maps $L(\mathbb{Z} / p, n) \rightarrow *$ for every $n \geqslant 0$. The fibrant replacement functor $R^{p}$ of $[\mathbf{1 6}]$ yields a rigid version of the pro- $p$-finite-completion of Artin-Mazur and Sullivan.

An extended discussion of this comparison and further aspects of profinite completion of spaces can be found in $[\mathbf{2 1}]$.

\subsection{Completion of spaces versus completion of groups}

Beside the completion functor of spaces $(\hat{\cdot)}: \mathcal{S} \rightarrow \hat{\mathcal{S}}$ there is a well-known profinite completion functor for discrete groups. For lack of better notation we will also denote the profinite completion of a group $G$ by $\hat{G}$. It is equipped with a natural homomorphism $G \rightarrow \hat{G}$ which is universal among continuous homomorphisms from $G$ to profinite groups.

Given a pointed space $X \in \mathcal{S}_{*}$, the homotopy groups of Definition 2.8 of its profinite completion $\hat{X} \in \hat{\mathcal{S}}_{*}$ are profinite groups. Hence the induced map $\pi_{t} X \rightarrow \pi_{t} \hat{X}$ factors through the group completion of $\pi_{t} X$, i.e., there is a commutative diagram

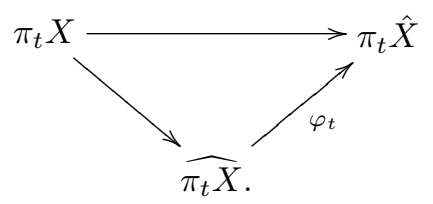

It is a fundamental question if completion of spaces and groups commute. Unfortunately, $\varphi_{t}$ is not an isomorphism in general. A similar phenomenon is well-known for group completion and cohomology. In [26], this led Serre to call a group $G$ good if the induced map $\psi: H^{*}(\hat{G} ; M) \rightarrow H^{*}(G ; M)$ between continuous and discrete group cohomology is an isomorphism for every finite $G$-module $M$. It turns out that after modifying slightly the notion of good groups by considering the action of the fundamental group on the higher homotopy groups, one gets a sufficient condition such that the completion of spaces commutes with the one of groups. 
Following [27], for a pointed space $X$, we call $\pi_{1}:=\pi_{1} X$ a good fundamental group, if it is a good group in the sense of Serre above such that

$$
H^{i}\left(\hat{\pi}_{1} ; M\right) \cong H^{i}\left(\pi_{1} ; M\right)
$$

is a finite group for all finite $\pi_{1}$-modules $M$ and all $i \geqslant 0$.

Let $\pi_{n}:=\pi_{n} X, n \geqslant 2$, be a higher homotopy group of $X$. It carries a canonical action of $\pi_{1}$. Let $\mathcal{P}$ be the filtered set of finite $\pi_{1}$-quotients of $\pi_{n}$. We denote by $\hat{\pi}_{n}^{\pi_{1}}:=\lim _{Q \in \mathcal{P}} Q$ the $\pi_{1}$-completion of $\pi_{n}$. This is, in particular, a profinite group on which $\pi_{1}$ acts continuously. The $\pi_{1}$-module $\pi_{n}$ is called a good higher homotopy group if

$$
H^{i}\left(\pi_{n} ; A\right) \cong H^{i}\left(\hat{\pi}_{n}^{\pi_{1}} ; A\right)
$$

is an isomorphism of finite groups for all finite coefficient groups $A$ and all $i \geqslant 0$. With these definitions we reinterpret the following result of Sullivan [27], Theorem 3.1 .

Theorem 2.15. Let $X$ be a connected pointed space.

(a) The canonical map $\varphi_{1}: \widehat{\pi_{1} X} \rightarrow \pi_{1} \hat{X}$ is an isomorphism of profinite groups, i.e., $\pi_{1} X \rightarrow \pi_{1} \hat{X}$ equals group completion.

(b) If $X$ has a good fundamental group and good higher homotopy groups, then $\varphi_{t}: \widehat{\pi_{t} X} \rightarrow \pi_{t} \hat{X}$ is an isomorphism of profinite groups for every $t$.

Proof. The first assertion follows immediately from the definition of the profinite fundamental group of a profinite space via profinite covering spaces with finite fibers and the analogous description of the usual fundamental group of $X$ (see [19]). The second assertion follows from Theorem 3.1 in $[\mathbf{2 7}]$ together with the fact discussed above that $|R \hat{X}|$ is a rigid model for the profinite completion of Sullivan, i.e., that $|R \hat{X}|$ is canonically isomorphic to $\hat{X}^{\mathrm{Su}}$ in $\mathcal{H}$.

\subsection{A concrete profinite replacement functor}

The result of Sullivan shows that profinite completion of spaces with finite homotopy groups induces a weak equivalence of underlying spaces. For a slightly smaller class of spaces, we have a more concrete description of a profinite model.

We recall that a space $X$ is called simple if it has an abelian fundamental group and if the action of the fundamental group on the higher homotopy groups is trivial.

Lemma 2.16. Let $X$ be a connected simple pointed space whose homotopy groups are all finite. Then there is a pointed simplicial finite set which is fibrant in $\hat{\mathcal{S}}_{*}$ and a pointed map $X \rightarrow F X$ which is a weak equivalence of underlying simplicial sets. In particular, it induces an isomorphism $\pi_{*} X \cong \pi_{*} F X$ of homotopy groups of the underlying simplicial sets. 
The assignment $X \mapsto F X$ is functorial in the sense that given a map $g: X \rightarrow Y$ between connected simple pointed spaces with finite homotopy groups, there is a map $F(g)$ in $\hat{\mathcal{S}}_{*}$ such that the following diagram of underlying simplicial sets commutes

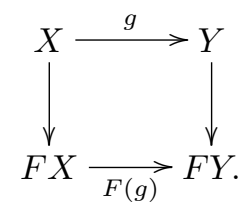

Proof. We can assume that $X$ is a fibrant simplicial set. Let $\pi_{n}:=\pi_{n} X$ be the $n$th finite homotopy group of $X$. We define the pointed profinite space $F X$ as the limit in $\hat{\mathcal{S}}_{*}$ of a specific Postnikov tower $\{X(n)\}_{n \geqslant 1}$ of $X$. Let $\operatorname{cosk}_{n} X \in \mathcal{S}_{*}$ be the $n$th coskeleton of $X$. It is equipped with natural pointed maps $X \rightarrow \operatorname{cosk}_{n} X$ and $\operatorname{cosk}_{n+1} X \rightarrow \operatorname{cosk}_{n} X$ for each $n \geqslant 0$. The fibrant pointed space $X$ is isomorphic to the $\operatorname{limit} \lim _{n} \operatorname{cosk}_{n} X$. Moreover, the map $\operatorname{cosk}_{n} X \rightarrow \operatorname{cosk}_{n-1} X$ sits in a homotopy pullback square in $\mathcal{S}_{*}$

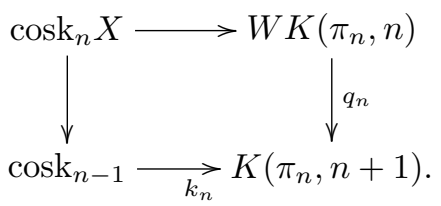

The map $q_{n}$ is the universal bundle over the simplicial group $K\left(\pi_{n}, n+1\right)$. For a simplicial group $\Gamma$, the contractible space $W \Gamma$ is defined by

$$
(W \Gamma)_{n}=\Gamma_{n} \times \Gamma_{n-1} \times \cdots \times \Gamma_{0} .
$$

The map $k_{n}$ is the $k$-invariant defined by a class

$$
\left[k_{n}\right] \in H^{n+1}\left(\operatorname{cosk}_{n-1} X ; \pi_{n}\right)
$$

in the cohomology of $\operatorname{cosk}_{n-1} X$ with coefficients in $\pi_{n}$.

Now we define the pointed profinite spaces $X(n)$ together with pointed maps $j_{n}: \operatorname{cosk}_{n} X \rightarrow X(n)$ which are weak equivalences of underlying pointed simplicial sets. For $n=1$, we define $X(1):=B \pi_{1} X$. It is a pointed simplicial finite set and a fibrant object in $\hat{\mathcal{S}}_{*}$. Choosing any weak equivalence $\operatorname{cosk}_{1} X \rightarrow B \pi_{1} X$ of simplicial sets provides a map $j_{1}: \operatorname{cosk}_{1} X \rightarrow X(1)$.

For $n \geqslant 2$, assume we are given $X(n-1)$ and a map $j_{n-1}: \operatorname{cosk}_{n-1} \rightarrow X(n-1)$. Up to homotopy there is a factorization in the category of pointed simplicial sets

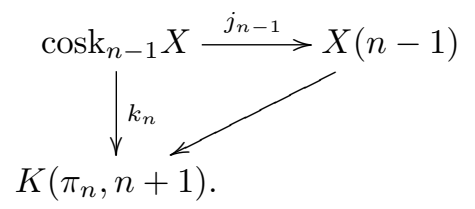

The space $X(n)$ and the map $X(n) \rightarrow X(n-1)$ is then defined as the pullback of 
the diagram of simplicial finite sets

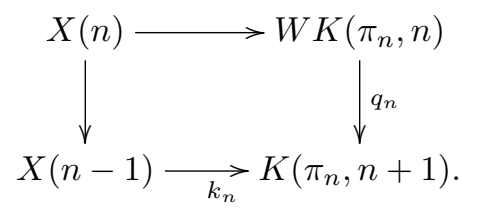

Since $\pi_{n}$ is a finite group, $K\left(\pi_{n}, n+1\right)$ and $W K\left(\pi_{n}, n\right)$ are finite in each degree. Moreover, the map

$$
q_{n}: W K\left(\pi_{n}, n\right) \rightarrow K\left(\pi_{n}, n+1\right)
$$

is a fibration in $\hat{\mathcal{S}}_{*}$ by $[\mathbf{2 1}]$, Theorem 3.7. Hence the pullback of (4) can be constructed in $\hat{\mathcal{S}}_{*}$ and $X(n)$ is a simplicial finite set which is a fibrant object in $\hat{\mathcal{S}}_{*}$. Since the map

$$
\operatorname{cosk}_{n} X \rightarrow W K\left(\pi_{n}, n\right) \times_{K\left(\pi_{n}, n+1\right)} \operatorname{cosk}_{n-1} X
$$

is a weak equivalence, the induced map $j_{n}: \operatorname{cosk}_{n} X \rightarrow X(n)$ is a weak equivalence of underlying simplicial sets. We define $F X$ to be the limit

$$
F X:=\lim _{n} X(n) .
$$

Since the finite set of $m$-simplices of $X(n)$ is isomorphic to the finite set of $m$ simplices of $X(n-1)$ for $m \leqslant n-1, F X$ is a simplicial finite set, and $F X$ is a fibrant object in $\hat{\mathcal{S}}_{*}$, since it is the limit of a tower of fibrations in $\hat{\mathcal{S}}_{*}$. Since the two maps $X \rightarrow \lim _{n} \operatorname{cosk}_{n} X$ and $\lim _{n} \operatorname{cosk}_{n} X \rightarrow \lim _{n} X(n)$ are weak equivalences of underlying simplicial sets, the associated map $\varphi: X \rightarrow F X$ is a weak equivalence of underlying simplicial sets and hence induces an isomorphism $\pi_{*} X \cong \pi_{*} F X$. Finally, we deduce the last assertion of the lemma from the fact that all the constructions involved in order to define $F X$ are functorial.

Lemma 2.16 provides a functorial construction $F$ that associates a weakly equivalent profinite space to a connected simple pointed space with finite homotopy groups. Since we also want to apply this construction to spectra, we need to understand its behavior with respect to taking loop spaces.

\section{Lemma 2.17.}

(a) Let $X$ and $Y$ be connected simple pointed spaces with finite homotopy groups and let $f: X \rightarrow Y$ be a weak equivalence of pointed simplicial sets. Then the induced map $F(f)$ is a weak equivalence in $\hat{\mathcal{S}}_{*}$.

(b) Let $W$ be a connected simple pointed space with finite homotopy groups. Then there is a natural weak equivalence $F(\Omega X) \stackrel{\sim}{\rightarrow} \Omega(F X)$ in $\hat{\mathcal{S}}_{*}$.

(c) If $V \rightarrow \Omega W$ is a weak equivalence between connected simple pointed spaces with finite homotopy groups, then the composite map $F V \rightarrow F(\Omega W) \rightarrow \Omega(F W)$ is a weak equivalence in $\hat{\mathcal{S}}_{*}$.

Proof. (a) Let $\{X(n)\}_{n}$ and $\{Y(n)\}_{n}$ be the two towers of fibrations corresponding to $X$ and $Y$, respectively, used in the proof of Lemma 2.16 in order to construct $F X$ and $F Y$. For each $n$, the map $X(n) \rightarrow Y(n)$ of simplicial finite sets induced by $f$ is a 
weak equivalence in $\mathcal{S}_{*}$ and in $\hat{\mathcal{S}}_{*}$. Hence the map of limits of the towers of fibrations

$$
F X=\lim _{n} X(n) \rightarrow \lim _{n} Y(n)=F Y
$$

is a weak equivalence in $\hat{\mathcal{S}}_{*}$ as well.

(b) This follows now from the fact that the map $K(\pi, n) \rightarrow \Omega(K(\pi, n+1))$ is a weak equivalence in $\mathcal{S}_{*}$ and $\hat{\mathcal{S}}_{*}$ for every finite group $\pi$ and every $n$.

(c) This is a consequence of (a) and (b).

\section{Profinite $G$-spaces}

\subsection{The simplicial model category of profinite $G$-spaces}

Let $G$ be a fixed profinite group. Let $S$ be a profinite set on which $G$ acts continuously, i.e., the group $G$ is acting on $S$ via a continuous map $\mu: G \times S \rightarrow S$. In this situation we say that $S$ is a profinite $G$-set. If $X$ is a profinite space and $G$ acts continuously on each $X_{n}$ such that the action is compatible with the face and degeneracy maps, then we call $X$ a profinite $G$-space. We denote by $\hat{\mathcal{S}}_{G}$ the category of profinite $G$-spaces. The morphism are given by $G$-equivariant maps of profinite spaces.

While a discrete $G$-space $Y$ is characterized as the colimit over the fixed point spaces $Y^{U}$ over all open subgroups, a profinite $G$-space $\mathrm{X}$ is the limit over its orbit spaces $X_{U}$. More explicitly, for an open subgroup $U$ of $G$, let $X_{U}$ be the quotient space under the action by $U$, i.e., the quotient $X / \sim$ with $x \sim y$ in $X$ if both are in the same orbit under $U$. It is easy to show that the canonical map $\phi: X \rightarrow \lim _{U} X_{U}$ is a homeomorphism, where $U$ runs through the open subgroups of $G$.

Moreover, we have the following lemma which was proven in [21], Lemma 4.2.

Lemma 3.1. Let $G$ be a profinite group and $X$ a profinite $G$-space. Then $X$ has a decomposition

$$
X=\lim _{Q_{G} \in \mathcal{R}_{G}(X)} X / Q_{G}
$$

in $\hat{\mathcal{S}}_{G}$ as an inverse limit of simplicial finite $G$-quotient sets where $\mathcal{R}_{G}(X)$ denotes the set of $G$-invariant simplicial open equivalence relations of $X$.

The category of profinite $G$-spaces has a simplicial structure. Let $X$ and $Y$ be profinite $G$-spaces. The mapping space $\operatorname{map}_{\hat{\mathcal{S}}_{G}}(X, Y)$ is defined as the simplicial set whose set of $n$-simplices is given as the set of $G$-equivariant maps

$$
\operatorname{map}_{\hat{\mathcal{S}}_{G}}(X, Y)_{n}=\operatorname{Hom}_{\hat{\mathcal{S}}_{G}}(X \times \Delta[n], Y)
$$

where $\Delta[n]$ has trivial $G$-action. This defines a functor

$$
\operatorname{map}_{\hat{\mathcal{S}}_{G}}(-,-): \hat{\mathcal{S}}_{G}^{\mathrm{op}} \times \hat{\mathcal{S}}_{G} \rightarrow \mathcal{S} \text {. }
$$

Let $K$ be a finite simplicial set, and $X$ a profinite $G$-space. We consider $K$ as a profinite $G$-space with trivial $G$-action. The tensor object $X \otimes K \in \hat{\mathcal{S}}_{G}$ is defined as the product $X \times K$ in $\hat{\mathcal{S}}_{G}$ with the diagonal $G$-action. The function object in $\hat{\mathcal{S}}_{G}$ is defined as the profinite $G$-space $\operatorname{hom}_{\hat{\mathcal{S}}_{G}}(K, X) \in \hat{\mathcal{S}}_{G}$ whose set of $n$-simplices is given by the profinite set of maps

$$
\operatorname{hom}_{\hat{\mathcal{S}}_{G}}(K, X)_{n}=\operatorname{Hom}_{\hat{\mathcal{S}}}(K \times \Delta[n], X)
$$

on which $G$ acts continuously via its continuous action on the target $X$. 
If $K$ is an arbitrary simplicial set, isomorphic to the colimit $\operatorname{colim}_{\alpha} K_{\alpha}$ of its finite simplicial subsets $K_{\alpha}$, and $X$ a profinite $G$-space, the tensor object $X \otimes K$ is the colimit in $\hat{\mathcal{S}}_{G}$ of the profinite $G$-spaces $X \times K_{\alpha}$.

The function object $\operatorname{hom}_{\hat{\mathcal{S}}_{*}}(K, X)$ is defined to be the limit in $\hat{\mathcal{S}}_{*}$ of the pointed profinite spaces $\operatorname{hom}_{\hat{\mathcal{S}}_{*}}\left(K_{\alpha}, X\right)$.

Let $X$ and $Y$ be profinite $G$-spaces and let $K$ be a simplicial set. Then mapping space, tensor and function objects are connected by the natural bijections

$$
\operatorname{map}_{\hat{\mathcal{S}}_{G}}(X \otimes K, Y) \cong \operatorname{map}_{\mathcal{S}}\left(K, \operatorname{map}_{\hat{\mathcal{S}}_{G}}(X, Y)\right)
$$

and

$$
\operatorname{map}_{\hat{\mathcal{S}}_{G}}\left(Y, \operatorname{hom}_{\hat{\mathcal{S}}_{G}}(K, X)\right) \cong \operatorname{map}_{\mathcal{S}}\left(K, \operatorname{map}_{\hat{\mathcal{S}}_{G}}(X, Y)\right) .
$$

This defines the structure of a simplicial category on $\hat{\mathcal{S}}_{G}$.

In order to get a model structure on $\hat{\mathcal{S}}_{G}$ one can find explicit sets of generating fibrations and trivial fibrations. They arise naturally by considering continuous $G$-actions on the corresponding generating sets for the model structure on $\hat{\mathcal{S}}$. The following result was proven in $[\mathbf{2 0}]$.

Theorem 3.2. There is a fibrantly generated left proper simplicial model structure on the category of profinite $G$-spaces such that a map $f$ is a weak equivalence (fibration) in $\hat{\mathcal{S}}_{G}$ if and only if its underlying map is a weak equivalence (fibration) in $\hat{\mathcal{S}}$. A map $f: X \rightarrow Y$ is a cofibration in $\hat{\mathcal{S}}_{G}$ if and only if $f$ is a levelwise injection, and the action of $G$ on $Y_{n}-f\left(X_{n}\right)$ is free for each $n \geqslant 0$. We denote its homotopy category by $\hat{\mathcal{H}}_{G}$.

Let $\hat{\mathcal{S}}_{* G}$ be the category of pointed profinite $G$-spaces. The objects of $\hat{\mathcal{S}}_{* G}$ are profinite $G$-spaces that are equipped with a base point that is fixed under $G$. The morphisms in $\hat{\mathcal{S}}_{* G}$ are the morphisms of profinite $G$-spaces that preserve the base points. Let $X$ and $Y$ be pointed profinite $G$-spaces. The smash product $X \wedge Y$ is again a pointed profinite $G$-space on which $G$ acts via the diagonal action. The mapping space $\operatorname{map}_{\hat{\mathcal{S}}_{*}}(X, Y)$ is defined as the simplicial set whose set of $n$-simplices is given as the set of maps

$$
\operatorname{map}_{\hat{\mathcal{S}}_{* G}}(X, Y)_{n}=\operatorname{Hom}_{\hat{\mathcal{S}}_{* G}}\left(X \wedge \Delta[n]_{+}, Y\right)
$$

where $\Delta[n]_{+}$is considered as a pointed profinite $G$-space with trivial $G$-action. This defines a functor

$$
\operatorname{map}_{\hat{\mathcal{S}}_{* G}}(-,-): \hat{\mathcal{S}}_{* G}^{\mathrm{op}} \times \hat{\mathcal{S}}_{* G} \rightarrow \mathcal{S} .
$$

Let $K$ be a finite simplicial set and $X$ a pointed profinite $G$-space. The tensor object $X \otimes K \in \hat{\mathcal{S}}_{* G}$ is defined as the smash product $X \wedge K_{+}$where $K_{+}$is considered as a pointed profinite $G$-space with trivial $G$-action. The function object in $\hat{\mathcal{S}}_{* G}$ is defined as the pointed profinite $G$-space $\operatorname{hom}_{\hat{\mathcal{S}}_{* G}}(K, X) \in \hat{\mathcal{S}}_{* G}$ whose set of $n$-simplices is given by the profinite set of maps

$$
\operatorname{hom}_{\hat{\mathcal{S}}_{* G}}(K, X)_{n}=\operatorname{Hom}_{\hat{\mathcal{S}}_{*}}\left(K_{+} \wedge \Delta[n]_{+}, X\right)
$$

on which $G$ acts continuously via its action on the target $X$. 
If $K$ is an arbitrary simplicial set isomorphic to the colimit colim $K_{\alpha}$ of its finite simplicial subsets $K_{\alpha}$, and $X$ a pointed profinite $G$-space, we define the tensor object $X \otimes K$ to be the colimit in $\hat{\mathcal{S}}_{* G}$ of the pointed profinite $G$-spaces $X \otimes K_{\alpha}$.

The function object $\operatorname{hom}_{\hat{\mathcal{S}}_{* G}}(K, X)$ is defined to be the limit in $\hat{\mathcal{S}}_{* G}$ of the pointed profinite $G$-spaces $\operatorname{hom}_{\hat{\mathcal{S}}_{* G}}\left(K_{\alpha}^{* G}, X\right)$.

Let $X$ and $Y$ be pointed profinite $G$-spaces and let $K$ be a simplicial set. Then mapping space, tensor and function objects are connected by the natural bijections

$$
\operatorname{map}_{\hat{\mathcal{S}}_{* G}}(X \otimes K, Y) \cong \operatorname{map}_{\mathcal{S}}\left(K, \operatorname{map}_{\hat{\mathcal{S}}_{* G}}(X, Y)\right)
$$

and

$$
\operatorname{map}_{\hat{\mathcal{S}}_{* G}}\left(Y, \operatorname{hom}_{\hat{\mathcal{S}}_{* G}}(K, X)\right) \cong \operatorname{map}_{\mathcal{S}}\left(K, \operatorname{map}_{\hat{\mathcal{S}}_{* G}}(X, Y)\right) .
$$

Again, if the finite simplicial set $K$ is already equipped with a base point and $X$ is a pointed profinite $G$-space, we also denote by $\operatorname{hom}_{\hat{\mathcal{S}}_{* G}}(K, X) \in \hat{\mathcal{S}}_{* G}$ the pointed profinite $G$-space whose set of $n$-simplices is given by the profinite set of maps

$$
\operatorname{hom}_{\hat{\mathcal{S}}_{* G}}(K, X)_{n}=\operatorname{Hom}_{\hat{\mathcal{S}}_{*}}\left(K \wedge \Delta[n]_{+}, X\right)=\lim _{\alpha} \operatorname{Hom}_{\hat{\mathcal{S}}_{*}}\left(K_{\alpha} \wedge \Delta[n]_{+}, X\right)
$$

on which $G$ acts continuously via its action on the target.

Example 3.3. We consider the simplicial circle $S^{1}$ as a pointed simplicial finite set with trivial $G$-action. Taking the smash product with $S^{1}$ defines a functor $\hat{\mathcal{S}}_{* G} \rightarrow \hat{\mathcal{S}}_{* G}$, $X \mapsto S^{1} \wedge X$. It is left adjoint to the functor $\hat{\mathcal{S}}_{* G} \rightarrow \hat{\mathcal{S}}_{* G}$ defined by sending a pointed profinite $G$-space $X$ to the function object $\operatorname{hom}_{\hat{\mathcal{S}}_{* G}}\left(S^{1}, X\right)$ in $\hat{\mathcal{S}}_{* G}$. In particular, the profinite loop space $\Omega X$ inherits from $X$ the structure as a pointed profinite $G$-space.

As in the nonequivariant case, the category of pointed profinite $G$-spaces inherits a model structure from $\hat{\mathcal{S}}_{G}$, since $\hat{\mathcal{S}}_{* G}$ is an under-category of $\hat{\mathcal{S}}_{G}$.

Theorem 3.4. The category $\hat{\mathcal{S}}_{* G}$ has the structure of a fibrantly generated left proper simplicial model category for which a map is a weak equivalence (cofibration, fibration) if and only if its underlying unpointed map is a weak equivalence (cofibration, fibration) in $\hat{\mathcal{S}}_{G}$. We denote the homotopy category by $\hat{\mathcal{H}}_{* G}$.

Remark 3.5. Let $X$ be a cofibrant pointed profinite $G$-space. As discussed in Remark 2.7, the pointed profinite $G$-space $X \wedge \Delta[1]_{+}$is a cylinder object for $X$ in $\hat{\mathcal{S}}_{* G}$. For the maps $X \rightarrow X \wedge \Delta[1]_{+}$are trivial cofibrations in $\hat{\mathcal{S}}_{* G}$, and the map $X \wedge \Delta[1]_{+} \rightarrow X$ is a weak equivalence in $\hat{\mathcal{S}}_{* G}$. Hence if $X$ is a cofibrant pointed profinite $G$-space and $Y$ is a fibrant pointed profinite $G$-space, there is a natural bijection

$$
\pi_{0} \operatorname{map}_{\hat{\mathcal{S}}_{* G}}(X, Y) \cong \operatorname{Hom}_{\hat{\mathcal{H}}_{* G}}(X, Y) .
$$

Since weak equivalences and fibrations in $\hat{\mathcal{S}}_{G}$ are determined by their underlying maps in $\hat{\mathcal{S}}$, one can hope that the fibrant replacement functor constructed for $\hat{\mathcal{S}}$ above also yields a fibrant replacement in $\hat{\mathcal{S}}_{G}$. In fact, this is the case. The point is that the loop groups and loop groupoids are free. If $G$ acts continuously on a profinite set $S$, then $G$ also acts continuously on the free profinite group on this set. Hence the construction of the fibrant replacement functor in $\hat{\mathcal{S}}_{G}$ is the same as for $\hat{\mathcal{S}}$. We just use Lemma 3.1 above to decompose a profinite $G$-space $X$ into a limit of simplicial finite $G$-sets. With a suitable notion of continuous group action on a groupoid we obtain a $G$-equivariant fibrant replacement in $\hat{\mathcal{S}}_{G}$ (for more details see [21], $§ 4.4$ ). 
Since we will be working with pointed profinite $G$-spaces, we would also like to have a fibrant replacement in $\hat{\mathcal{S}}_{* G}$. For a pointed profinite $G$-space $X \in \hat{\mathcal{S}}_{* G}$, the basepoint $p: * \rightarrow X$ and the decomposition of $X$ as a $\operatorname{limit} X \cong \lim _{Q_{G}} X / Q_{G}$ as pointed simplicial finite $G$-sets provides each $X / Q_{G}$ with a choice of basepoint which is compatible in the system $\left\{X / Q_{G}\right\}$. Then $[\mathbf{2 1}]$, Theorem 4.12, implies the following pointed version.

Theorem 3.6. Let $G$ be a profinite group and let $X$ be a pointed profinite $G$-space. The map

$$
\eta: X \rightarrow \bar{W} \hat{\Gamma} X=\lim _{Q_{G} \in \mathcal{R}_{G}(X)} \bar{W} \hat{\Gamma}\left(X / Q_{G}\right)
$$

is a weak equivalence in $\hat{\mathcal{S}}_{* G}$. The pointed profinite $G$-space $\bar{W} \hat{\Gamma} X$ is fibrant in $\hat{\mathcal{S}}_{* G}$. Hence $\eta$ defines a functorial fibrant replacement in $\hat{\mathcal{S}}_{* G}$. Moreover, $X$ is weakly equivalent in $\hat{\mathcal{S}}_{* G}$ to a limit of simplicial finite $G$-sets which are fibrant in $\hat{\mathcal{S}}_{* G}$

$$
X \simeq \lim _{Q_{G}, U_{Q_{G}}} \bar{W}\left(\Gamma\left(X / Q_{G}\right)\right) / U_{Q_{G}}
$$

where $U_{Q_{G}}$ runs through the $G$-invariant normal subgroupoids of $\Gamma\left(X / Q_{G}\right)$ such that the quotient $\Gamma\left(X / Q_{G}\right) / U_{Q_{G}}$ is a simplicial finite groupoid

\subsection{Homotopy groups of pointed profinite $G$-spaces}

Definition 3.7. Let $X$ be a pointed profinite $G$-space and let $R_{G} X$ be a fibrant replacement of $X$ in the model structure on $\hat{\mathcal{S}}_{* G}$. Then we define the $n$th profinite homotopy group of $X$ for $n \geqslant 2$ to be the group

$$
\pi_{n}(X):=\pi_{0}\left(\Omega^{n}\left(R_{G} X\right)\right) .
$$

Remark 3.8. Let $X$ be a pointed profinite $G$-space. Since weak equivalences and fibrations in $\hat{\mathcal{S}}_{* G}$ are determined by the underlying maps in $\hat{\mathcal{S}}_{*}$, the underlying homotopy groups of $X$ of Definition 3.7 coincide with the homotopy groups of Definition 2.8 of $X$ after forgetting the $G$-action on $X$.

Moreover, the homotopy groups of Definition 3.7 coincide with the homotopy groups of the underlying fibrant pointed simplicial set $\left|R_{G} X\right|$.

Proposition 3.9. Let $X$ be a pointed profinite $G$-space. The set of connected components of $X$ is a profinite $G$-set. The profinite fundamental group of $X$ is a profinite $G$-group. The nth homotopy group of $X$ is a profinite $G$-module for $n \geqslant 2$.

Proof. Let $R_{G}$ be the functorial fibrant replacement functor of Theorem 3.6. Since

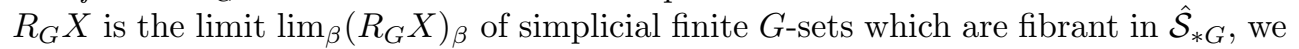
obtain that

$$
\operatorname{hom}_{\hat{\mathcal{S}}_{* G}}\left(S^{1}, R_{G} X\right)=\operatorname{hom}_{\hat{\mathcal{S}}_{* G}}\left(S^{1}, \lim _{\beta}\left(R_{G} X\right)_{\beta}\right)=\lim _{\beta} \operatorname{hom}_{\hat{\mathcal{S}}_{* G}}\left(S^{1},\left(R_{G} X\right)_{\beta}\right)
$$

is a cofiltered limit of fibrant simplicial finite $G$-sets. Moreover, the underlying profinite space of $\operatorname{hom}_{\hat{\mathcal{S}}_{* G}}\left(S^{1}, R_{G} X\right)$ is equal to the fibrant profinite space $\operatorname{hom}_{\hat{\mathcal{S}}_{*}}\left(S^{1}, R_{G} X\right)$. Hence in order to prove the assertion, it suffices by Proposition 2.11 to show the assertions for a simplicial finite $G$-set which is fibrant in $\hat{\mathcal{S}}_{* G}$.

So we can assume that $X$ is a simplicial finite $G$-set which is fibrant in $\hat{\mathcal{S}}_{* G}$. The set $X_{0}$ of 0 -simplices and the set $X_{1}$ of 1 -simplices of $X$ are finite discrete $G$-sets. 
Hence they can be written as colimits of the fixed points under the open subgroups of $G$, i.e.,

$$
X_{0}=\operatorname{colim}_{U} X_{0}^{U}, X_{1}=\operatorname{colim}_{U} X_{1}^{U}
$$

where $U$ runs through the open subgroups of $G$. The set $\pi_{0} X$ of connected components is defined as the colimit of the diagram

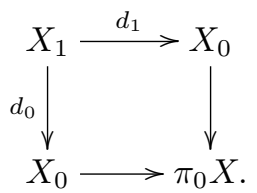

Since $\pi_{0}$ commutes with filtered colimits, we also get $\pi_{0} X=\operatorname{colim}_{U} \pi_{0}\left(X^{U}\right)$. Since $X$ is a fibrant simplicial finite set and since the fixed points can be viewed as a cofiltered limit, Proposition 2.11 shows that we have $\pi_{0}\left(X^{U}\right) \cong\left(\pi_{0} X\right)^{U}$. Hence we obtain

$$
\pi_{0} X=\operatorname{colim}_{U}\left(\pi_{0} X\right)^{U}
$$

i.e., that $G$ acts continuously on the finite discrete set $\pi_{0} X$. Since $\operatorname{hom}_{\hat{\mathcal{S}}_{* G}}\left(S^{1}, X\right)$ is a fibrant simplicial finite $G$-set if $X$ is a fibrant simplicial finite $G$-set, the assertions on the fundamental group and the higher homotopy groups follow from the result on $\pi_{0}$.

\subsection{Homotopy limits of pointed profinite $G$-spaces}

Homotopy limits of diagrams of pointed profinite $G$-spaces can be constructed in the same way as for pointed profinite spaces. Let $I$ be a small category and let $X(-): I \rightarrow \hat{\mathcal{S}}_{* G}$ be a functor from $I$ to pointed profinite $G$-spaces. We define the pointed profinite $G$-space $\operatorname{hom}_{\hat{\mathcal{S}}_{* G}}(B(I /-), X(-))$ as the equalizer in $\hat{\mathcal{S}}_{* G}$ of the diagram of pointed profinite $G$-spaces

$$
\prod_{i \in I} \operatorname{hom}_{\hat{\mathcal{S}}_{* G}}(B(I / i), X(i)) \rightrightarrows \prod_{\alpha: i \rightarrow i^{\prime} \in I} \operatorname{hom}_{\hat{\mathcal{S}}_{* G}}\left(B(I / i), X\left(i^{\prime}\right)\right) .
$$

Definition 3.10. Let $I$ be a small category and $X(-): I \rightarrow \hat{\mathcal{S}}_{* G}$ be a functor. The homotopy limit of $X(-)$ is defined to be the pointed profinite $G$-space

$$
\operatorname{holim}_{i \in I} X(i):=\operatorname{hom}_{\hat{\mathcal{S}}_{* G}}(B(I /-), X(-)) \text {. }
$$

Lemma 3.11. Let $X(-)$ be a functor from a small category $I$ to pointed profinite $G$-spaces. If $X(i)$ is fibrant for every $i \in I$, then $\operatorname{holim}_{i \in I} X(i)$ is fibrant in $\hat{\mathcal{S}}_{* G}$. Let $f(-): X(-) \rightarrow Y(-)$ be a natural transformation of functors from the small category $I$ to the full subcategory of fibrant pointed profinite G-spaces. Let

$$
f:=\operatorname{holim}_{i \in I} f(i): \operatorname{holim}_{i \in I} X(i) \rightarrow \operatorname{holim}_{i \in I} Y(i)
$$

be the induced map on homotopy limits. If $f(i): X(i) \rightarrow Y(i)$ is a weak equivalence in $\hat{\mathcal{S}}_{* G}$ for every $i \in I$, then $f$ is a weak equivalence in $\hat{\mathcal{S}}_{* G}$.

Proof. As for pointed profinite spaces, this follows from [12], Theorem 19.4.1 and Theorem 19.4.2. 
Lemma 3.12. Let $X(-): I \rightarrow \hat{\mathcal{S}}_{* G}$ be a small cofiltering diagram such that each $X(i)$ is connected and fibrant in $\hat{\mathcal{S}}_{* G}$. Then the natural isomorphism of homotopy groups of underlying simplicial sets

$$
\pi_{q}\left(\left|\operatorname{holim}_{i \in I} X(i)\right|\right) \cong \lim _{i \in I} \pi_{q}(|X(i)|)
$$

of Lemma 2.14 is an isomorphism of profinite G-groups for $q \geqslant 0$.

Proof. The construction of the spectral sequence computing the homotopy groups of the homotopy limit is functorial. Hence the isomorphism of Lemma 2.14 for the underlying diagram of pointed profinite spaces is in fact a $G$-equivariant isomorphism of profinite $G$-groups.

\subsection{A concrete equivariant profinite replacement}

In this section we discuss a functor that sends certain $G$-spaces to profinite $G$ spaces. We call a simplicial set $X$ a $G$-space, if $X$ is a simplicial object in the category of $G$-sets for the abstract underlying group of $G$. In order to simplify the argument and in order to make the construction more concrete we will make two assumptions, on the one hand that the profinite group $G$ is strongly complete and on the other hand that the spaces have finite homotopy groups.

A profinite group $G$ is called strongly complete in [25], if every subgroup of finite index is open in $G$, or, in other words, if $G=\hat{G}$ as profinite groups. The profinite completion of an abstract group is itself strongly complete. But in general there are subgroups of finite index which are not open in the given topology. The significance of this property for us, is that for a strongly complete profinite group $G$, every finite set $S$ with a $G$-action is a continuous discrete $G$-set.

Serre conjectured that every topologically finitely generated profinite group $G$ is strongly complete, where topologically finitely generated means that $G$ contains a dense subgroup which is finitely generated as a group. He showed this conjecture for finitely generated pro- $p$-groups. Recently, Nikolov and Segal proved the full conjecture for every finitely generated profinite group in [17]. The proof relies on the classification of finite simple groups. The most important examples of stronlgy complete profinite groups for us are provided by the extended Morava stabilizer group $G_{n}$ and all of its closed subgroups (see e.g. [5], p. 330).

The following lemma is an equivariant version of Lemma 2.16 which is of fundamental importance for this paper.

Lemma 3.13. Let $G$ be a strongly complete profinite group and $X$ be a connected simple pointed $G$-space whose homotopy groups are all finite. Then there is a simplicial finite $G$-set $F_{G} X$ which is a fibrant object in $\hat{\mathcal{S}}_{* G}$ and a natural $G$-equivariant pointed map $\varphi: X \rightarrow F_{G} X$ which is a weak equivalence of underlying simplicial sets. In particular, it induces an isomorphism $\pi_{*} X \cong \pi_{*} F_{G} X$ of homotopy groups of the underlying simplicial sets.

The assignment $X \mapsto F_{G} X$ is functorial in the sense that given a $G$-equivariant map $h: X \rightarrow Y$ between connected simple pointed simplicial $G$-sets with finite homotopy groups, there is a map $F(h)$ in $\hat{\mathcal{S}}_{* G}$ such that the following diagram of underlying 
simplicial G-sets commutes

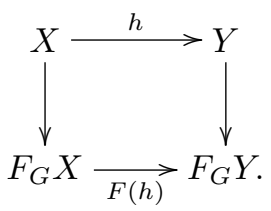

Proof. Let $X \rightarrow X^{\prime}$ be a functorial fibrant replacement in $\mathcal{S}_{*}$. The map $X \rightarrow X^{\prime}$ is $G$-equivariant and a weak equivalence of underlying simplicial sets. Hence we can assume that the underlying simplicial set of $X$ is a fibrant object in $\mathcal{S}_{*}$.

Let $\pi_{n}:=\pi_{n} X$ be the $n$th finite homotopy group of $X$. Since $X$ is a $G$-space, each $\pi_{n}$ is a $G$-module. Moreover, since $G$ is strongly complete, $G$ acts continuously on each finite discrete group $\pi_{n}$. We define the profinite $G$-space $F_{G} X$ again as the limit in $\hat{\mathcal{S}}_{* G}$ of a specific Postnikov tower $\{X(n)\}_{n \geqslant 1}$ of $X$. Let $\operatorname{cosk}_{n} X \in \mathcal{S}_{*}$ be the $n$th coskeleton of $X$. Since $\operatorname{cosk}_{n}$ is a functor, the space $\operatorname{cosk}_{n} X$ is a simplicial $G$ set and the associated natural pointed maps $X \rightarrow \operatorname{cosk}_{n} X$ and $\operatorname{cosk}_{n} X \rightarrow \operatorname{cosk}_{n-1} X$ are $G$-equivariant for each $n \geqslant 0$. Moreover, the map $\operatorname{cosk}_{n} X \rightarrow \operatorname{cosk}_{n-1} X$ sits in a homotopy pullback square of $G$-equivariant maps

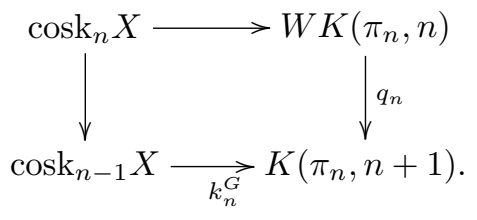

The $\operatorname{map} k_{n}^{G}$ is the $G$-equivariant $k$-invariant defined by a class

$$
\left[k_{n}^{G}\right] \in H_{G}^{n+1}\left(\operatorname{cosk}_{n-1} X ; \pi_{n}\right)
$$

in the $G$-equivariant cohomology of $\operatorname{cosk}_{n-1} X$ (see e.g. [10], pp. 207-208).

Now we define profinite $G$-spaces $X(n)$ together with $G$-equivariant pointed maps $j_{n}: \operatorname{cosk}_{n} X \rightarrow X(n)$ which are weak equivalences of underlying pointed simplicial sets. For $n=1$, we define $X(1):=B \pi_{1} X$. It is a pointed simplicial finite $G$-set and a fibrant object in $\hat{\mathcal{S}}_{* G}$. Choosing any $G$-equivariant map $\operatorname{cosk}_{1} X \rightarrow B \pi_{1} X$ which is a weak equivalence of simplicial sets provides a map $j_{1}: \operatorname{cosk}_{1} X \rightarrow X(1)$. (This $G$ equivariant choice is possible, since the category of simplicial $G$-sets, for the underlying abstract group of $G$, can be equipped with a model structure in which weak equivalences and fibrations are determined by their underlying non-equivariant maps.)

For $n \geqslant 2$, assume we are given $X(n-1)$ and a map $j_{n-1}: \operatorname{cosk}_{n-1} \rightarrow X(n-1)$. Up to $G$-equivariant homotopy there is a factorization in the category of pointed simplicial $G$-sets

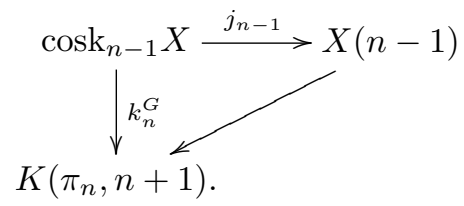

The space $X(n)$ and the map $X(n) \rightarrow X(n-1)$ is then defined as the pullback of 
the diagram of simplicial finite $G$-sets

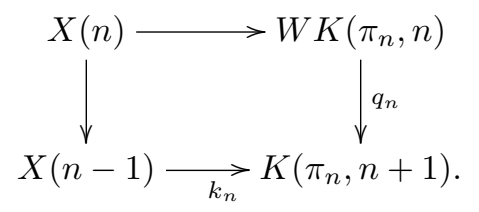

Since $\pi_{n}$ is a finite $G$-module, the spaces $K\left(\pi_{n}, n+1\right)$ and $W K\left(\pi_{n}, n\right)$ are simplicial finite $G$-sets. Moreover, since $G$ is strongly complete, the action of $G$ on all the finite sets involved is continuous and the map

$$
q_{n}: W K\left(\pi_{n}, n\right) \rightarrow K\left(\pi_{n}, n+1\right)
$$

is a fibration in $\hat{\mathcal{S}}_{* G}$ by Theorem 3.4, and [21] Proposition 3.7. Hence the pullback of (6) can be constructed in $\hat{\mathcal{S}}_{* G}$ and $X(n)$ is a simplicial finite discrete $G$-set which is a fibrant object in $\hat{\mathcal{S}}_{* G}$.

Since the map

$$
\operatorname{cosk}_{n} X \rightarrow W K\left(\pi_{n}, n\right) \times_{K\left(\pi_{n}, n+1\right)} \operatorname{cosk}_{n-1} X
$$

is a weak equivalence, we obtain an induced weak equivalence $j_{n}: \operatorname{cosk}_{n} X \rightarrow X(n)$ of underlying simplicial sets. Now we can define $F_{G} X$ to be the limit

$$
F_{G} X:=\lim _{n} X(n) \text {. }
$$

Since the finite discrete $G$-set of $m$-simplices of $X(n)$ is isomorphic to the finite discrete $G$-set of $m$-simplices of $X(n-1)$ for $m \leqslant n-1, F_{G} X$ is a simplicial object of finite discrete $G$-sets. Moreover, $F_{G} X$ is a fibrant object in $\hat{\mathcal{S}}_{* G}$, since it is the limit of a tower of fibrations in $\hat{\mathcal{S}}_{* G}$. Furthermore, since the $G$-equivariant maps $X \rightarrow \lim _{n} \operatorname{cosk}_{n} X$ and $\lim _{n} \operatorname{cosk}_{n} X \rightarrow \lim _{n} X(n)$ are weak equivalences of underlying simplicial sets, the associated map $\varphi: X \rightarrow F_{G} X$, which is functorial and hence $G$-equivariant, is a weak equivalence of underlying simplicial sets. In particular, it induces an isomorphism $\pi_{*} X \cong \pi_{*} F_{G} X$.

The functoriality follows as in the non-equivariant case from the fact that all constructions used to define $F_{G} X$ are functorial.

As in the non-equivariant case, we have to know how $F_{G}$ behaves with respect to taking loop spaces.

Lemma 3.14. Let $G$ be a strongly complete profinite group.

(a) Let $X$ and $Y$ be connected simple pointed simplicial $G$-sets with finite homotopy groups and let $f: X \rightarrow Y$ be a G-equivariant map that is a weak equivalence of underlying pointed simplicial sets. Then the induced map $F_{G}(f)$ is a weak equivalence in $\hat{\mathcal{S}}_{* G}$.

(b) Let $W$ be a connected simple pointed simplicial $G$-set with finite homotopy groups. Then there is a natural weak equivalence $F_{G}(\Omega X) \stackrel{\sim}{\rightarrow} \Omega\left(F_{G} X\right)$ in $\hat{\mathcal{S}}_{* G}$.

(c) If $V \rightarrow \Omega W$ is a $G$-equivariant map between connected simple pointed simplicial $G$-sets with finite homotopy groups which is a weak equivalence of underlying simplicial sets, then the composite map $F_{G} V \rightarrow F_{G}(\Omega W) \rightarrow \Omega\left(F_{G} W\right)$ is a weak equivalence in $\hat{\mathcal{S}}_{* G}$. 
Proof. Since weak equivalences in $\hat{\mathcal{S}}_{* G}$ are determined by their underlying maps in $\hat{\mathcal{S}}_{*}$, the lemma follows from the corresponding results in Lemma 2.17 and the construction of $F_{G}$.

\section{Stable profinite homotopy theory}

\subsection{Profinite spectra}

Now we want to stabilize the model structure of profinite spaces. Since the simplicial circle $S^{1}=\Delta^{1} / \partial \Delta^{1}$ is a simplicial finite set and hence an object in $\hat{\mathcal{S}}_{*}$, we may stabilize $\hat{\mathcal{S}}_{*}$ by considering sequences of pointed profinite spaces together with connecting maps for the suspension.

Definition 4.1. A profinite spectrum $X$ consists of a sequence of pointed profinite spaces $X_{n} \in \hat{\mathcal{S}}_{*}$ and maps $\sigma_{n}: S^{1} \wedge X_{n} \rightarrow X_{n+1}$ in $\hat{\mathcal{S}}_{*}$ for $n \geqslant 0$. A morphism $f: X \rightarrow Y$ of profinite spectra consists of maps $f_{n}: X_{n} \rightarrow Y_{n}$ in $\hat{\mathcal{S}}_{*}$ for $n \geqslant 0$ such that $\sigma_{n}\left(1 \wedge f_{n}\right)=f_{n+1} \sigma_{n}$. We denote by $\operatorname{Sp}\left(\hat{\mathcal{S}}_{*}\right)$ the corresponding category of profinite spectra.

As for profinite spaces, the word "profinite" in the term "profinite spectrum" does not mean that we look at inverse systems of finite spectra in the usual sense. Instead we look at spectra that are built out of simplicial profinite sets.

The category of profinite spectra is a simplicial category. Let $X$ and $Y$ be profinite spectra. The mapping space $\operatorname{map}_{\operatorname{Sp}\left(\hat{\mathcal{S}}_{*}\right)}(X, Y)$ is defined as the simplicial set whose set of $n$-simplices is given as the set of maps

$$
\operatorname{map}_{\mathrm{Sp}\left(\hat{\mathcal{S}}_{*}\right)}(X, Y)_{n}=\operatorname{Hom}_{\mathrm{Sp}\left(\hat{\mathcal{S}}_{*}\right)}\left(X \wedge \Delta[n]_{+}, Y\right)
$$

where $X \wedge \Delta[n]_{+}$is the levelwise smash product of $X$ with the pointed simplicial finite set $\Delta[1]_{+}$. This defines a functor

$$
\operatorname{map}_{\mathrm{Sp}\left(\hat{\mathcal{S}}_{*}\right)}(-,-): \operatorname{Sp}\left(\hat{\mathcal{S}}_{*}\right)^{\text {op }} \times \operatorname{Sp}\left(\hat{\mathcal{S}}_{*}\right) \rightarrow \mathcal{S} .
$$

Let $K$ be a simplicial set, and $X$ a profinite spectrum. The tensor object $X \otimes K \in$ $\operatorname{Sp}\left(\hat{\mathcal{S}}_{*}\right)$ is defined as the spectrum whose $n$th pointed profinite space is $X_{n} \wedge K_{+}$. The function object in $\operatorname{Sp}\left(\hat{\mathcal{S}}_{*}\right)$ is defined as the profinite spectrum $\operatorname{hom}_{\operatorname{Sp}\left(\hat{\mathcal{S}}_{*}\right)}(K, X) \in$ $\operatorname{Sp}\left(\hat{\mathcal{S}}_{*}\right)$ whose $n$th pointed profinite space is given by

$$
\left(\operatorname{hom}_{\mathrm{Sp}\left(\hat{\mathcal{S}}_{*}\right)}(K, X)\right)_{n}=\operatorname{hom}_{\hat{\mathcal{S}}_{*}}\left(K, X_{n}\right) .
$$

Recall that the structure $S^{1} \wedge X_{n} \rightarrow X_{n+1}$ is determined by its adjoint

$$
X_{n} \rightarrow \Omega X_{n+1} \text {. }
$$

Hence the structure map of $\operatorname{hom}_{\mathrm{Sp}\left(\hat{\mathcal{S}}_{*}\right)}(K, X)$ is determined by the map

$$
\operatorname{hom}_{\hat{\mathcal{S}}_{*}}\left(K, X_{n}\right) \rightarrow \operatorname{hom}_{\hat{\mathcal{S}}_{*}}\left(K, \Omega X_{n+1}\right) \cong \Omega\left(\operatorname{hom}_{\hat{\mathcal{S}}_{*}}\left(K, X_{n+1}\right)\right)
$$

given by adjunction (1). 
Let $X$ and $Y$ be profinite spectra and let $K$ be a simplicial set. We have the natural bijections

$$
\operatorname{map}_{\mathrm{Sp}\left(\hat{\mathcal{S}}_{*}\right)}(X \otimes K, Y) \cong \operatorname{map}_{\mathcal{S}}\left(K, \operatorname{map}_{\mathrm{Sp}\left(\hat{\mathcal{S}}_{*}\right)}(X, Y)\right)
$$

and

$$
\operatorname{map}_{\mathrm{Sp}\left(\hat{\mathcal{S}}_{*}\right)}\left(Y, \operatorname{hom}_{\mathrm{Sp}\left(\hat{\mathcal{S}}_{*}\right)}(K, X)\right) \cong \operatorname{map}_{\mathcal{S}}\left(K, \operatorname{map}_{\mathrm{Sp}\left(\hat{\mathcal{S}}_{*}\right)}(X, Y)\right) .
$$

If the simplicial set $K$ is already equipped with a base point and $X$ is a profinite spectrum, we also denote by $\operatorname{hom}_{\mathrm{Sp}\left(\hat{\mathcal{S}}_{*}\right)}(K, X) \in \mathrm{Sp}\left(\hat{\mathcal{S}}_{*}\right)$ the profinite spectrum whose $n$th space is $\operatorname{hom}_{\hat{\mathcal{S}}_{*}}\left(K, X_{n}\right)$.

Observing that the functor $\operatorname{Sp}\left(\hat{\mathcal{S}}_{*}\right) \rightarrow \operatorname{Sp}\left(\hat{\mathcal{S}}_{*}\right), X \mapsto S^{1} \wedge X$ is a left Quillen endofunctor, the stable model structure on $\operatorname{Sp}\left(\hat{\mathcal{S}}_{*}\right)$ is constructed as in [18] in two steps as a dual version of the stabilization techniques in [14]. First we need a projective model structure.

Definition 4.2. A map $f$ in $\operatorname{Sp}\left(\hat{\mathcal{S}}_{*}\right)$ is a projective weak equivalence (projective fibration) if each map $f_{n}$ is a weak equivalence (fibration) in $\hat{\mathcal{S}}_{*}$. A map $i$ is a projective cofibration if it has the left lifting property with respect to all projective trivial fibrations.

The following proposition is proved as in [14], Proposition 1.14.

Proposition 4.3. A map $i: A \rightarrow B$ in $\mathrm{Sp}\left(\hat{\mathcal{S}}_{*}\right)$ is a projective (trivial) cofibration if and only if $i_{0}: A_{0} \rightarrow B_{0}$ and the induced maps $j_{n}: A_{n} \amalg_{S^{1} \wedge A_{n-1}} S^{1} \wedge B_{n-1} \rightarrow B_{n}$ for $n \geqslant 1$ are (trivial) cofibrations in $\hat{\mathcal{S}}_{*}$.

Proposition 4.4. The projective weak equivalences, projective fibrations and projective cofibrations define a left proper fibrantly generated simplicial model structure on $\operatorname{Sp}\left(\hat{\mathcal{S}}_{*}\right)$.

Proof. That we obtain a left proper fibrantly generated model structure can be proven in essentially the same way as Theorem 1.13 in [14]. In order to show the factorization axiom one uses a cosmall object argument and the fact that $\hat{\mathcal{S}}_{*}$ is fibrantly generated. It remains to prove that this model structure is simplicial. We have defined mapping spaces and tensor and cotensor objects for $\operatorname{Sp}\left(\hat{\mathcal{S}}_{*}\right)$ above. Let $i: A \rightarrow B$ be a cofibration of simplicial sets and $p: X \rightarrow Y$ a projective fibration in $\operatorname{Sp}\left(\hat{\mathcal{S}}_{*}\right)$. We have to show that the induced map

$$
\left(i^{*}, p_{*}\right): \operatorname{hom}_{\mathrm{Sp}\left(\hat{\mathcal{S}}_{*}\right)}(B, X) \rightarrow \operatorname{hom}_{\mathrm{Sp}\left(\hat{\mathcal{S}}_{*}\right)}(A, X) \times_{\operatorname{hom}_{\mathrm{Sp}\left(\hat{\mathcal{S}}_{*}\right)}(A, Y)} \operatorname{hom}_{\mathrm{Sp}\left(\hat{\mathcal{S}}_{*}\right)}(B, Y)
$$

is a projective fibration in $\operatorname{Sp}\left(\hat{\mathcal{S}}_{*}\right)$, which is trivial if either $i$ or $p$ is trivial. For $n \geqslant 0$, the $n$th map $\left(i^{*}, p_{*}\right)_{n}$ is given by the map of pointed profinite spaces

$$
\left(i^{*}, p_{n *}\right): \operatorname{hom}_{\hat{\mathcal{S}}_{*}}\left(B, X_{n}\right) \rightarrow \operatorname{hom}_{\hat{\mathcal{S}}_{*}}\left(A, X_{n}\right) \times_{\operatorname{hom}_{\hat{\mathcal{S}}_{*}}\left(A, Y_{n}\right)} \operatorname{hom}_{\hat{\mathcal{S}}_{*}}\left(B, Y_{n}\right) .
$$

The model structure on $\hat{\mathcal{S}}_{*}$ is simplicial. This implies that $\left(i^{*}, p_{n *}\right)$ is a fibration, since $i$ is a cofibration and $p_{n}$ is a fibration. Moreover, $\left(i^{*}, p_{n *}\right)$ is a weak equivalence if either $i$ or $p_{n}$ is a weak equivalence. Since projective weak equivalences and projective fibrations are determined levelwise, this shows that $\left(i^{*}, p_{*}\right)$ is a projective fibration which is a trivial projective fibration if either $i$ or $p$ is trivial. 
In the second step we enlarge the class of weak equivalences by localizing the projective model structure further.

Definition 4.5. A profinite spectrum $E \in \operatorname{Sp}\left(\hat{\mathcal{S}}_{*}\right)$ is called an $\Omega$-spectrum if each $E_{n}$ is fibrant in $\hat{\mathcal{S}}_{*}$ and the adjoint structure maps

$$
E_{n} \rightarrow \Omega E_{n+1}=\operatorname{hom}_{\hat{\mathcal{S}}_{*}}\left(S^{1}, E_{n+1}\right)
$$

are weak equivalences in $\hat{\mathcal{S}}_{*}$ for all $n \geqslant 0$.

A map $f: X \rightarrow Y$ of profinite spectra is called

- a (stable) equivalence if any projective cofibrant replacement $Q f: Q X \rightarrow Q Y$ in $\operatorname{Sp}\left(\hat{\mathcal{S}}_{*}\right)$ induces a weak equivalence of mapping spaces

$$
\operatorname{map}_{\mathrm{Sp}\left(\hat{\mathcal{S}}_{*}\right)}(Q Y, E) \rightarrow \operatorname{map}_{\mathrm{Sp}\left(\hat{\mathcal{S}}_{*}\right)}(Q X, E)
$$

for all $\Omega$-spectra $E$;

- a (stable) cofibration if and only if it is a projective cofibration;

- a (stable) fibration if it has the right lifting property with respect to all maps that are stable equivalences and stable cofibrations.

The following result was shown in [19], Theorem 2.36. The proof is based on the dual of the general stabilization machinery for model structures by Hovey [14] and the localization for fibrantly generated simplicial model structures in [18], Theorem 6 (see also [18], Theorem 14).

Theorem 4.6. The classes of stable equivalences, stable fibrations and stable cofibrations define a stable simplicial model structure on profinite spectra. The fibrant objects in $\operatorname{Sp}\left(\hat{\mathcal{S}}_{*}\right)$ are exactly the $\Omega$-spectra. A map in $\operatorname{Sp}\left(\hat{\mathcal{S}}_{*}\right)$ between $\Omega$-spectra is a stable equivalence if and only if it is a projective weak equivalence. We denote the corresponding homotopy category by $\hat{\mathcal{S H}}$. In particular, the functor $S^{1} \wedge \cdot: \hat{\mathcal{S H}} \rightarrow \hat{\mathcal{S H}}$ is an equivalence of categories.

Let $\operatorname{Sp}\left(\mathcal{S}_{*}\right)$ be the stable model category of Bousfield-Friedlander spectra of [2].

Proposition 4.7. The levelwise profinite completion functor

$$
(\hat{\cdot}): \operatorname{Sp}\left(\mathcal{S}_{*}\right) \rightarrow \operatorname{Sp}\left(\hat{\mathcal{S}}_{*}\right)
$$

preserves cofibrations and stable equivalences between cofibrant objects. The forgetful functor

$$
|\cdot|: \operatorname{Sp}\left(\hat{\mathcal{S}}_{*}\right) \rightarrow \operatorname{Sp}\left(\mathcal{S}_{*}\right)
$$

preserves stable fibrations and weak equivalences between fibrant objects. In particular, $(\hat{.})$ and $|\cdot|$ induce derived functors on the homotopy categories and the pair $((\hat{\cdot}),|\cdot|)$ is a Quillen pair of adjoint functors.

Proof. Let $i: A \rightarrow B$ be a cofibration in $\operatorname{Sp}\left(\mathcal{S}_{*}\right)$. Since $(\hat{)}): \mathcal{S}_{*} \rightarrow \hat{\mathcal{S}}_{*}$ preserves cofibrations and pushouts as a left Quillen functor, the maps $\hat{i}_{0}$ and $\hat{j}_{n}$ are cofibrations in $\hat{\mathcal{S}}_{*}$. Hence $\hat{i}$ is a cofibration in $\operatorname{Sp}\left(\hat{\mathcal{S}}_{*}\right)$. 
Let $Y$ be a pointed profinite space. As a right adjoint the forgetful functor for pointed profinite spaces $|\cdot|: \hat{\mathcal{S}}_{*} \rightarrow \mathcal{S}_{*}$ commutes with cotensors. Hence we have

$$
\left|\operatorname{hom}_{\hat{\mathcal{S}}_{*}}\left(S^{1}, Y\right)\right|=\operatorname{hom}_{\mathcal{S}_{*}}\left(S^{1},|Y|\right) \text {. }
$$

Moreover, $|\cdot|: \hat{\mathcal{S}}_{*} \rightarrow \mathcal{S}_{*}$ sends fibrant pointed profinite spaces to fibrant pointed spaces and preserves weak equivalences between fibrant objects. Hence if $E$ is an $\Omega$-spectrum in $\operatorname{Sp}\left(\hat{\mathcal{S}}_{*}\right)$, then $|E|$ is an $\Omega$-spectrum in $\operatorname{Sp}\left(\mathcal{S}_{*}\right)$.

Let $f: X \rightarrow Y$ be a stable equivalence between cofibrant objects in $\operatorname{Sp}\left(\mathcal{S}_{*}\right)$ and let $E$ be an $\Omega$-spectrum in $\operatorname{Sp}\left(\hat{\mathcal{S}}_{*}\right)$. Since profinite completion is left adjoint to the forgetful functor we get a commutative diagram of simplicial sets

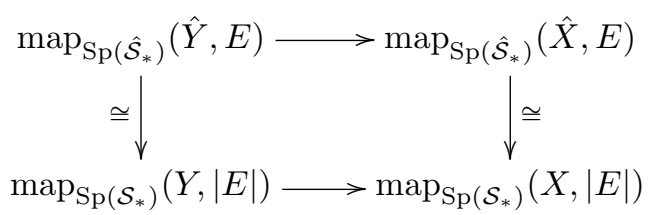

whose vertical maps are isomorphisms. Since the map $f$ is a stable equivalence between cofibrant objects in $\operatorname{Sp}\left(\mathcal{S}_{*}\right)$ it induces a weak equivalence of mapping spaces

$$
\operatorname{map}_{\mathrm{Sp}\left(\mathcal{S}_{*}\right)}(Y, F) \rightarrow \operatorname{map}_{\mathrm{Sp}\left(\mathcal{S}_{*}\right)}(X, F)
$$

for every $\Omega$-spectrum $F$ in $\operatorname{Sp}\left(\mathcal{S}_{*}\right)$. (This follows for example from [14], Corollary 3.5.) Since $|E|$ is an $\Omega$-spectrum in $\operatorname{Sp}\left(\mathcal{S}_{*}\right)$, this shows that the lower horizontal map in (7) is a weak equivalence. Since the vertical maps are isomorphisms, this implies that the upper horizontal map in (7) is a weak equivalence as well. Since this holds for every $\Omega$-spectrum $E$ in $\operatorname{Sp}\left(\hat{\mathcal{S}}_{*}\right)$, the map $\hat{f}$ is a stable equivalence in $\operatorname{Sp}\left(\hat{\mathcal{S}}_{*}\right)$. The same argument shows that $(\hat{.})$ preserves trivial cofibrations.

Since the forgetful functor is the right adjoint of profinite completion, this implies that $|\cdot|$ preserves fibrations and stable equivalences between fibrant objects.

\subsection{Homotopy groups of profinite spectra}

For a positive integer $k \geqslant 0$, there is an evaluation functor

$$
\operatorname{Ev}_{k}: \operatorname{Sp}\left(\hat{\mathcal{S}}_{*}\right) \rightarrow \hat{\mathcal{S}}_{*}, X \mapsto X_{k},
$$

sending a profinite spectrum $X$ to its $k$ th pointed profinite space $X_{k}$. It is a right Quillen functor for the stable model structure on $\operatorname{Sp}\left(\hat{\mathcal{S}}_{*}\right)$. Its left Quillen adjoint is the functor $\Sigma^{k}(-): \hat{\mathcal{S}}_{*} \rightarrow \operatorname{Sp}\left(\hat{\mathcal{S}}_{*}\right)$ defined by

$$
\begin{gathered}
\left(\Sigma^{k} Y\right)_{m}=S^{m-k} Y \text { if } m \geqslant k \text { and } \\
\left(\Sigma^{k} Y\right)_{m}=* \text { otherwise }
\end{gathered}
$$

where $S^{m-k} Y$ denotes the pointed profinite space obtained by smashing the pointed profinite space $Y(m-k)$-times with $S^{1}$.

Let $S^{0}$ be the discrete pointed simplicial finite set generated by two points as 0simplices one of which is the basepoint. We define the sphere spectrum $\mathbb{S}^{0}$ to be the profinite spectrum $\Sigma^{0}\left(S^{0}\right)$ whose $m$ th pointed profinite space is $S^{m}$, i.e., $S^{1}$ smashed with itself $m$-times. 
For a negative integer $n<0$, we define the $n$th suspension $\mathbb{S}^{n}$ of the sphere spectrum to be the profinite spectrum $\Sigma^{-n}\left(S^{0}\right)$. Its $m$ th space is $S^{m+n}$ if $m \geqslant-n$ and just a point otherwise.

For a positive integer $n>0$, we define the $n$th suspension $\mathbb{S}^{n}$ of the sphere spectrum to be the profinite spectrum $\Sigma^{0}\left(S^{n}\right)$ whose $m$ th space is $S^{m+n}$ for every $m \geqslant 0$.

Via the model structure of Theorem 4.6 we can define homotopy groups of a profinite spectrum $X$. Let $R X$ denote a functorial fibrant replacement of $X$ in the stable model structure on $\operatorname{Sp}\left(\hat{\mathcal{S}}_{*}\right)$ and, for an integer $n$, let $\mathbb{S}^{n}$ be the $n$th suspension of the sphere spectrum. We define $\pi_{n} X$ to be the group

$$
\pi_{n} X:=\operatorname{Hom}_{\mathcal{S} \mathcal{H}}\left(\mathbb{S}^{n}, R X\right) .
$$

\section{Proposition 4.8.}

(a) Let $X$ be a fibrant profinite spectrum, i.e., an $\Omega$-spectrum in $\operatorname{Sp}\left(\hat{\mathcal{S}}_{*}\right)$, and $n \in \mathbb{Z}$. Let $k \geqslant 0$ be any natural number such that $n+k \geqslant 0$. Then there is a natural isomorphism of groups

$$
\pi_{n} X \cong \pi_{n+k} X_{k}
$$

where $\pi_{n+k} X_{k}$ denotes the profinite homotopy group of the $k$ th pointed profinite space of $X$.

(b) The homotopy groups of a fibrant profinite spectrum $X$ are isomorphic to the homotopy groups of the underlying $\Omega$-spectrum $|X|$ in $\operatorname{Sp}\left(\mathcal{S}_{*}\right)$.

(c) The homotopy groups of a profinite spectrum are abelian profinite groups.

Proof. (a) If $n \geqslant 0$, then the Quillen adjoint pair $\left(\Sigma^{0}, \mathrm{Ev}_{0}\right)$ yields a natural isomorphism

$$
\pi_{n} X=\operatorname{Hom}_{\hat{\mathcal{H}} \mathcal{H}}\left(\mathbb{S}^{n}, X\right)=\operatorname{Hom}_{\hat{\mathcal{S H}}}\left(\Sigma^{0}\left(S^{n}\right), X\right) \cong \operatorname{Hom}_{\hat{\mathcal{H}}_{*}}\left(S^{n}, X_{0}\right) .
$$

The right hand side is naturally isomorphic to $\pi_{0}\left(\Omega^{n}\left(X_{0}\right)\right)=\pi_{n}\left(X_{0}\right)$. Since the maps $X_{0} \rightarrow \Omega^{k}\left(X_{k}\right)$ are weak equivalences of fibrant objects in $\hat{\mathcal{S}}_{*}$ for every $k \geqslant 0$, this proves the assertion for $n \geqslant 0$.

For $n<0$, the Quillen adjoint pair $\left(\Sigma^{-n}, \mathrm{Ev}_{-n}\right)$ yields a natural isomorphism

$$
\pi_{n} X=\operatorname{Hom}_{\hat{\mathcal{H}} \mathcal{H}}\left(\mathbb{S}^{n}, X\right)=\operatorname{Hom}_{\hat{\mathcal{H}} \mathcal{H}}\left(\Sigma^{-n}\left(S^{0}\right), X\right) \cong \operatorname{Hom}_{\hat{\mathcal{H}}_{*}}\left(S^{0}, X_{-n}\right) .
$$

The right hand side is naturally isomorphic to $\pi_{0}\left(X_{-n}\right)$. Reindexing and using the fact that $X$ is an $\Omega$-spectrum gives $\pi_{n} X=\pi_{n+k}\left(X_{k}\right)$ for any $k \geqslant 0$ such that $n+k \geqslant 0$. This finishes the proof of (a).

(b) Since the underlying spectrum $|X|$ is an $\Omega$-spectrum in $\operatorname{Sp}\left(\mathcal{S}_{*}\right)$, the $n$th homotopy group of $|X|$ is given by the abelian group $\pi_{n+k}\left(\left|X_{k}\right|\right)$ for any $k \geqslant 0$ such that $n+k \geqslant 0$. By Lemma 2.9, there is a natural isomorphism

$$
\pi_{n+k}\left(\left|X_{k}\right|\right) \cong \pi_{n+k}\left(X_{k}\right) .
$$

The assertion now follows from (a).

(c) This follows from the fact that the homotopy groups of a profinite space are profinite groups and that $\Omega^{2} Y$ is an abelian group object for any pointed profinite space $Y$. 
We can also define generalized cohomology groups with coefficients in profinite spectra. Let $X$ be a fibrant profinite spectrum. Let $Z$ be a spectrum in $\operatorname{Sp}\left(\mathcal{S}_{*}\right)$ which is isomorphic to a colimit of suspended sphere spectra $\mathbb{S}^{n_{\alpha}}$ for integers $n_{\alpha} \in \mathbb{Z}$ indexed by a filtered category. In this case, we can define the $k$ th generalized cohomology group of $Z$ with coefficients in $X$ to be the filtered limit

$$
X^{k} Z:=\lim _{\alpha} \operatorname{Hom}_{\mathcal{S} \mathcal{H}}\left(\mathbb{S}^{n_{\alpha}}, X[k]\right)
$$

where $X[k]$ denotes the $\Omega$-spectrum in $\operatorname{Sp}\left(\hat{\mathcal{S}}_{*}\right)$ whose $m$ th space is $X_{m-k}$ for $m \geqslant k$ and a point otherwise. Since each abelian group

$$
\operatorname{Hom}_{\hat{\mathcal{H}} \mathcal{H}}\left(\mathbb{S}^{n_{\alpha}}, X[k]\right)=\pi_{n_{\alpha}}(X[k])
$$

carries a profinite structure by Proposition 4.8, we deduce that the group $X^{k} Z$ is also a profinite abelian group.

Moreover, since each space in the sphere spectrum $\mathbb{S}^{n}$ is a pointed simplicial finite set for any integer $n$, the Quillen adjunction of Proposition 4.7 between profinite completion and the forgetful functor yields a natural isomorphism of abelian groups

$$
\operatorname{Hom}_{\mathcal{S} \mathcal{H}}\left(\mathbb{S}^{n}, X\right) \cong \operatorname{Hom}_{\mathcal{S H}}\left(\mathbb{S}^{n},|X|\right) .
$$

Hence the $k$ th generalized cohomology group of $Z$ with coefficients in the profinite spectrum $X$ coincides with the $k$ th generalized cohomology group of $Z$ with coefficients in the underlying $\Omega$-spectrum $|X|$ in $\operatorname{Sp}\left(\mathcal{S}_{*}\right)$, i.e., there is a natural isomorphism

$$
X^{k} Z=\lim _{\alpha} \operatorname{Hom}_{\mathcal{S} \mathcal{H}}\left(\mathbb{S}^{n_{\alpha}}, X[k]\right) \cong \lim _{\alpha} \operatorname{Hom}_{\mathcal{S H}}\left(\mathbb{S}^{n_{\alpha}},|X[k]|\right) \cong|X|^{k} Z
$$

\subsection{Homotopy limits of profinite spectra}

Let $I$ be a small category and let $X(-)$ be a functor from $I$ to the full subcategory of $\Omega$-spectra in $\operatorname{Sp}\left(\hat{\mathcal{S}}_{*}\right)$. For each $n \geqslant 0$ and each $i \in I$, we have a fibrant pointed profinite space $X_{n}(i):=X(i)_{n}$. So for every $n \geqslant 0$, this defines an $I$-diagram $X_{n}(-)$ of fibrant pointed profinite spaces. The homotopy $\operatorname{limit}_{\operatorname{holim}} \operatorname{li}_{i} X_{n}(i)$ in $\hat{\mathcal{S}}_{*}$ is again a fibrant pointed profinite space by Lemma 2.13 . Since the homotopy limit is defined using cotensors, there is a natural isomorphism

$$
\operatorname{holim}_{i \in I} \Omega\left(X_{n}(i)\right) \cong \Omega\left(\operatorname{holim}_{i \in I} X_{n}(i)\right) .
$$

Since each $X(i)$ is an $\Omega$-spectrum in $\operatorname{Sp}\left(\hat{\mathcal{S}}_{*}\right)$ and since holim ${ }_{i \in I}$ preserves weak equivalences between fibrant objects by Lemma 2.13 , for each $n \geqslant 0$ we obtain a weak equivalence in $\hat{\mathcal{S}}_{*}$

$$
\operatorname{holim}_{i \in I} X_{n}(i) \stackrel{\sim}{\rightarrow} \operatorname{holim}_{i \in I} \Omega X_{n}(i) \cong \Omega \operatorname{holim}_{i \in I} X_{n}(i) .
$$

Hence together with these structure maps the sequence of fibrant pointed profinite spaces $\operatorname{holim}_{i \in I} X_{n}(i)$ defines an $\Omega$-spectrum in $\operatorname{Sp}\left(\hat{\mathcal{S}}_{*}\right)$ that we denote by $\operatorname{holim}_{i \in I} X(i)$ and call the homotopy limit of the diagram $X(-)$ (see $[\mathbf{2 8}], \S 5$, for the analogous story for $\left.\operatorname{Sp}\left(\mathcal{S}_{*}\right)\right)$. 
Lemma 4.9. Let $f: X(-) \rightarrow Y(-)$ be a natural transformation of functors from a small category I to the full subcategory of $\Omega$-spectra in $\operatorname{Sp}\left(\hat{\mathcal{S}}_{*}\right)$. If $f(i): X(i) \rightarrow Y(i)$ is an equivalence in $\operatorname{Sp}\left(\hat{\mathcal{S}}_{*}\right)$ for every $i \in I$, then the induced map

$$
\operatorname{holim}_{i \in I} X(i) \rightarrow \operatorname{holim}_{i \in I} Y(i)
$$

is an equivalence of $\Omega$-spectra in $\operatorname{Sp}\left(\hat{\mathcal{S}}_{*}\right)$.

Proof. We already know that holim sends a small diagram of $\Omega$-spectra in $\operatorname{Sp}\left(\hat{\mathcal{S}}_{*}\right)$ to an $\Omega$-spectrum in $\operatorname{Sp}\left(\hat{\mathcal{S}}_{*}\right)$. Since holim is constructed termwise and since stable equivalences between $\Omega$-spectra are exactly the projective equivalences in $\operatorname{Sp}\left(\hat{\mathcal{S}}_{*}\right)$, the assertion follows from the corresponding result Lemma 2.13 for $\hat{\mathcal{S}}_{*}$.

Lemma 4.10. Let $X(-): I \rightarrow \operatorname{Sp}\left(\hat{\mathcal{S}}_{*}\right)$ be a small cofiltering diagram of $\Omega$-spectra in $\operatorname{Sp}\left(\hat{\mathcal{S}}_{*}\right)$. There is a natural isomorphism of homotopy groups of underlying spectra for every $q \in \mathbb{Z}$

$$
\pi_{q}\left(\left|\operatorname{holim}_{i \in I} X(i)\right|\right) \cong \lim _{i \in I} \pi_{q}(|X(i)|) .
$$

Proof. Since the profinite spectrum holim $i \in I X(i)$ is constructed levelwise and is an $\Omega$-spectrum in $\operatorname{Sp}\left(\hat{\mathcal{S}}_{*}\right)$, the assertion follows from Proposition 4.8 and Lemma 2.14.

\subsection{Spectra with finite homotopy groups}

The functors given by taking profinite completion of spectra and taking profinite completion of abelian groups are related to each other in a similar way as the corresponding profinite completion functor for spaces. For the purposes of this paper, the following concrete result will be sufficient.

Theorem 4.11. Let $X \in \operatorname{Sp}\left(\mathcal{S}_{*}\right)$ be a spectrum whose homotopy groups are all finite groups. Then there is a natural map

$$
X \rightarrow F^{s} X
$$

of spectra from $X$ to a profinite spectrum $F^{s} X$ built of simplicial finite sets such that $F^{s} X$ is fibrant in $\operatorname{Sp}\left(\hat{\mathcal{S}}_{*}\right)$ and $X \rightarrow F^{s} X$ is a stable equivalence of underlying spectra. In particular, it induces an isomorphism $\pi_{*} X \cong \pi_{*}\left|F^{s} X\right|$ of homotopy groups of underlying spectra.

The assignment $X \mapsto F^{s} X$ is functorial in the sense that given a map $g: X \rightarrow Y$ between spectra with finite homotopy groups, there is a map $F^{s}(g)$ in $\operatorname{Sp}\left(\hat{\mathcal{S}}_{*}\right)$ such that the following diagram of underlying spectra commutes

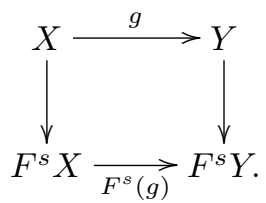

Proof. Let $X$ be a spectrum whose homotopy groups are finite. We can assume that $X$ is an $\Omega$-spectrum in $\operatorname{Sp}\left(\mathcal{S}_{*}\right)$, i.e., that each pointed space $X_{n}$ is fibrant in $\mathcal{S}_{*}$ and 
$X_{n} \rightarrow \Omega\left(X_{n+1}\right)=\operatorname{hom}_{\mathcal{S}_{*}}\left(S^{1}, X_{n+1}\right)$ is a weak equivalence for all $n \geqslant 0$. This implies for all $k \geqslant 0$

$$
\pi_{k}\left(X_{n}\right) \cong \pi_{k}\left(\Omega\left(X_{n+1}\right)\right) \cong \pi_{k+1}\left(X_{n+1}\right) .
$$

The $i$ th stable homotopy group of the spectrum $X$ is given as any of the isomorphic homotopy groups $\pi_{i+n}\left(X_{n}\right)$ for positive $n$ such that $i+n \geqslant 0$. Since the homotopy groups of $X$ are finite, each of the groups $\pi_{k}\left(X_{n}\right)$ is a finite group for all $k, n \geqslant 0$.

Since $X$ is a spectrum, we can assume that each $X_{n}$ is a connected simple pointed space. Hence we can apply the finite replacement $F$ of Lemma 2.16 to each space $X_{n}$ and obtain the profinite spectrum $F^{s} X$. By Lemma 2.17, the induced maps

$$
F X_{n} \rightarrow \Omega\left(F X_{n+1}\right)
$$

are weak equivalences in $\hat{\mathcal{S}}_{*}$. Since each $F X_{n}$ is fibrant in $\hat{\mathcal{S}}_{*}$, this implies that the levelwise fibrant profinite spectrum $F^{s} X$ is already an $\Omega$-spectrum in $\operatorname{Sp}\left(\hat{\mathcal{S}}_{*}\right)$. Furthermore, the $i$ th stable homotopy group of the profinite spectrum $F^{s} X$ is given by any of the isomorphic finite groups

$$
\pi_{i+n}\left(F X_{n}\right) \cong \pi_{i+n}\left(X_{n}\right)
$$

for positive $n$ such that $i+n \geqslant 0$. This shows that the map $X \rightarrow\left|F^{s} X\right|$ is a weak equivalence of underlying spectra and induces an isomorphism $\pi_{*} X \cong \pi_{*} F^{s} X$. Functoriality follows from the functoriality of $F$ in Lemma 2.16 .

\subsection{Profinite spectra versus pro-spectra}

In [4], Christensen and Isaksen develop a model structure on the category of prospectra. Unfortunately, the comparison between the category of pro-spectra and profinite spectra is not as straightforward as we would like it to be. First, Christensen and Isaksen use the category of symmetric spectra as a model for the stable homotopy category. Since the category of profinite spectra is based on a more naive notion of spectra, we have to limit our comparison with symmetric spectra to their underlying Bousfield-Friedlander spectra. Second, for an arbitrary profinite spectrum there is not a canonical way to write it as a cofiltered system of spectra. Nevertheless, we can define a functor from pro-spectra to profinite spectra as follows.

Let $\left\{X_{i}\right\}$ be a pro-spectrum. We denote by the same symbol its underlying proobject in the category $\operatorname{Sp}\left(\mathcal{S}_{*}\right)$ of Bousfield-Friedlander spectra. First we apply the (set-theoretic and levelwise) profinite completion functor $\operatorname{Sp}\left(\mathcal{S}_{*}\right) \rightarrow \operatorname{Sp}\left(\hat{\mathcal{S}}_{*}\right)$ to each spectrum in the system. This yields a pro-object of profinite spectra $\left\{\hat{X}_{i}\right\}$. Second we take a levelwise functorial fibrant replacement in $\operatorname{Sp}\left(\hat{\mathcal{S}}_{*}\right)$ and obtain the pro-object of profinite spectra $\left\{R \hat{X}_{i}\right\}$. In the final step we take the homotopy limit in $\operatorname{Sp}\left(\hat{\mathcal{S}}_{*}\right)$ of the cofiltered diagram underlying the pro-object $\left\{R \hat{X}_{i}\right\}$. By abuse of notation we denote the resulting profinite spectrum by $\hat{X}$.

As for pro-spaces, the comparison of the cofiltered system of homotopy groups $\left\{\pi_{k} X_{i}\right\}$ and the profinite homotopy group $\pi_{k} \hat{X}$ is difficult in general. But if the groups $\pi_{k} X_{i}$ are finitely generated for all $i$, then one can show, using the results stated in the second section, that $\pi_{k} \hat{X}$ is the profinite completion of the pro-group $\left\{\pi_{k} X_{i}\right\}$.

If a map $f:\left\{X_{i}\right\} \rightarrow\left\{Y_{i}\right\}$ of pro-spectra is a levelwise equivalence of BousfieldFriedlander spectra, then Proposition 4.7 and the invariance of homotopy limits show 
that the induced map $\hat{f}: \hat{X} \rightarrow \hat{Y}$ is a stable equivalence in $\operatorname{Sp}\left(\hat{\mathcal{S}}_{*}\right)$. But for a general $\pi^{*}$-equivalence $f$ of pro-spectra (see $\left.[4], \S 6\right) \hat{f}$ does not have to be an equivalence in $\operatorname{Sp}\left(\hat{\mathcal{S}}_{*}\right)$. Moreover, since the completion functor is a composition of profinite completion, which is a left adjoint, and the homotopy limit functor, it is neither a left nor a right adjoint functor.

We can modify the completion functor a little bit when we start with a restricted class of fibrant pro-spectra. In [4] a spectrum is called homotopy-finite if it is weakly equivalent to a finite complex. By [4], Proposition 6.8, the fibrant objects in the $\pi^{*}$-model structure on pro-spectra are the pro-spectra which are isomorphic to a pro-spectrum that is levelwise homotopy-finite and strictly fibrant. Let $\left\{X_{i}\right\}$ be a $\pi^{*}$ fibrant pro-spectrum. After possibly changing it by an isomorphism, we can assume that $\left\{X_{i}\right\}$ is a levelwise homotopy-finite spectrum. We assume moreover that the homotopy groups of each $X_{i}$ are all finite. In this case, we can apply the functor $F^{s}$ of Theorem 4.11 levelwise to obtain the pro-object $\left\{F^{s} X_{i}\right\}$ of $\Omega$-spectra in $\operatorname{Sp}\left(\hat{\mathcal{S}}_{*}\right)$. Taking the homotopy limit of the underlying diagram yields a profinite spectrum $\hat{X}^{F}$. By its construction, the functor $\left\{X_{i}\right\} \mapsto \hat{X}^{F}$ preserves fibrant objects. Moreover, since $\left\{\pi_{k} X_{i}\right\}$ is a cofiltered system of finite groups, which are preserved by $F^{s}$, the profinite homotopy groups $\pi_{*} \hat{X}^{F}$ are isomorphic in the category of pro-groups to $\left\{\pi_{*} X_{i}\right\}$.

\section{The equivariant profinite stable homotopy category}

\subsection{Profinite $G$-spectra}

Let $G$ be again a profinite group. We consider $S^{1}$ as a simplicial finite set with trivial $G$-action.

Definition 5.1. A profinite $G$-spectrum $X$ is a sequence of pointed profinite $G$ spaces $\left\{X_{n}\right\}$ together with maps $S^{1} \wedge X_{n} \rightarrow X_{n+1}$ of pointed profinite $G$-spaces for each $n \geqslant 0$. A map of profinite $G$-spectra $X \rightarrow Y$ is a collection of maps $X_{n} \rightarrow Y_{n}$ in $\hat{\mathcal{S}}_{* G}$ compatible with the structure maps of $X$ and $Y$. We denote the category of profinite $G$-spectra by $\operatorname{Sp}\left(\hat{\mathcal{S}}_{* G}\right)$.

In [20] a slightly different notion of profinite $G$-spectra was introduced by using a cofibrant replacement of $S^{1}$ in $\hat{\mathcal{S}}_{* G}$. This notion turns out to be less appropriate for our purposes. Therefore, we consider Definition 5.1 as the correct notion of profinite $G$-spectra.

The category of profinite $G$-spectra is a simplicial category. Let $X$ and $Y$ be profinite $G$-spectra. The mapping space $\operatorname{map}_{\operatorname{Sp}\left(\hat{\mathcal{S}}_{* G}\right)}(X, Y)$ is defined as the simplicial set whose set of $n$-simplices is given as the set of maps

$$
\operatorname{map}_{\mathrm{Sp}\left(\hat{\mathcal{S}}_{* G}\right)}(X, Y)_{n}=\operatorname{Hom}_{\mathrm{Sp}\left(\hat{\mathcal{S}}_{* G}\right)}\left(X \wedge \Delta[n]_{+}, Y\right)
$$

where $\Delta[n]_{+}$is considered as a simplicial finite $G$-set with trivial $G$-action. This defines a functor

$$
\operatorname{map}_{\operatorname{Sp}\left(\hat{\mathcal{S}}_{* G}\right)}(-,-): \operatorname{Sp}\left(\hat{\mathcal{S}}_{* G}\right)^{\text {op }} \times \operatorname{Sp}\left(\hat{\mathcal{S}}_{* G}\right) \rightarrow \mathcal{S} .
$$

Let $K$ be a simplicial set and $X$ a profinite $G$-spectrum. The tensor object $X \otimes K \in$ $\operatorname{Sp}\left(\hat{\mathcal{S}}_{* G}\right)$ is defined as the profinite $G$-spectrum whose $n$th pointed profinite $G$-space is $X_{n} \wedge K_{+}$, where we consider $K_{+}$as a pointed simplicial set with trivial $G$-action. The 
function object in $\operatorname{Sp}\left(\hat{\mathcal{S}}_{* G}\right)$ is defined as the profinite spectrum $\operatorname{hom}_{\operatorname{Sp}\left(\hat{\mathcal{S}}_{* G}\right)}(K, X) \in$ $\operatorname{Sp}\left(\hat{\mathcal{S}}_{* G}\right)$ whose $n$th pointed profinite $G$-space is given by

$$
\left(\operatorname{hom}_{\mathrm{Sp}\left(\hat{\mathcal{S}}_{* G}\right)}(K, X)\right)_{n}=\operatorname{hom}_{\hat{\mathcal{S}}_{* G}}\left(K, X_{n}\right) .
$$

The structure map of $\operatorname{hom}_{\mathrm{Sp}\left(\hat{\mathcal{S}}_{* G}\right)}(K, X)$ is determined by the $G$-equivariant map

$$
\operatorname{hom}_{\hat{\mathcal{S}}_{* G}}\left(K, X_{n}\right) \rightarrow \operatorname{hom}_{\hat{\mathcal{S}}_{* G}}\left(K, \Omega X_{n+1}\right) \cong \Omega\left(\operatorname{hom}_{\hat{\mathcal{S}}_{* G}}\left(K, X_{n+1}\right)\right) .
$$

Let $X$ and $Y$ be profinite $G$-spectra and let $K$ be a simplicial set. We have natural bijections

$$
\operatorname{map}_{\operatorname{Sp}\left(\hat{\mathcal{S}}_{* G}\right)}(X \otimes K, Y) \cong \operatorname{map}_{\mathcal{S}}\left(K, \operatorname{map}_{\mathrm{Sp}\left(\hat{\mathcal{S}}_{* G}\right)}(X, Y)\right)
$$

and

$$
\operatorname{map}_{\mathrm{Sp}\left(\hat{\mathcal{S}}_{* G}\right)}\left(Y, \operatorname{hom}_{\mathrm{Sp}\left(\hat{\mathcal{S}}_{* G}\right)}(K, X)\right) \cong \operatorname{map}_{\mathcal{S}}\left(K, \operatorname{map}_{\mathrm{Sp}\left(\hat{\mathcal{S}}_{* G}\right)}(X, Y)\right) .
$$

If the simplicial set $K$ is already equipped with a base point and $X$ is a profinite $G$-spectrum, we also denote by $\operatorname{hom}_{\operatorname{Sp}\left(\hat{\mathcal{S}}_{* G}\right)}(K, X) \in \operatorname{Sp}\left(\hat{\mathcal{S}}_{*}\right)$ the profinite $G$-spectrum whose $n$th space is $\operatorname{hom}_{\hat{\mathcal{S}}_{* G}}\left(K, X_{n}\right)$.

As for profinite spectra we would like to construct a stable model structure on $\operatorname{Sp}\left(\hat{\mathcal{S}}_{* G}\right)$. Therefore, we have to check the following lemma.

Lemma 5.2. The functor $X \mapsto S^{1} \wedge X$ is a left Quillen endofunctor of $\hat{\mathcal{S}}_{* G}$.

Proof. We saw in Example 3.3 that smashing with $S^{1}$ is a left adjoint functor whose right adjoint is given by the functor $\hat{\mathcal{S}}_{* G} \rightarrow \hat{\mathcal{S}}_{* G}, X \rightarrow \Omega X$. Since weak equivalences in $\hat{\mathcal{S}}_{* G}$ are determined by the underlying maps in $\hat{\mathcal{S}}_{*}$, it follows that the functor $X \mapsto S^{1} \wedge X$ from $\hat{\mathcal{S}}_{* G}$ to itself preserves weak equivalences. In order to see that it preserves cofibrations we recall that a map $f: X \rightarrow Y$ in $\hat{\mathcal{S}}_{* G}$ is a cofibration if and only if $f$ is a levelwise injection and the action of $G$ on $Y_{n}-f\left(X_{n}\right)$ is free for each $n \geqslant 0$. We know that the smash product preserves levelwise injections. Now $G$ acts diagonally on the smash product $S^{1} \wedge Y$. This implies that if the $G$-action is free on $Y_{n}-f\left(X_{n}\right)$, then the $G$-action on $\left(S^{1} \wedge Y\right)_{n}-\left(S^{1} \wedge f\right)\left(\left(S^{1} \wedge X\right)_{n}\right)$ is free as well. Hence $X \mapsto S^{1} \wedge X$ is a left adjoint endofunctor that preserves weak equivalences and cofibrations in $\hat{\mathcal{S}}_{* G}$.

As for profinite spectra, we start with a projective model structure.

Definition 5.3. A map $f$ in $\operatorname{Sp}\left(\hat{\mathcal{S}}_{* G}\right)$ is a projective weak equivalence (projective fibration) if each map $f_{n}$ is a weak equivalence (fibration) in $\hat{\mathcal{S}}_{* G}$. A map $i$ is a projective cofibration if it has the left lifting property with respect to all projective trivial fibrations.

The following proposition follows again as in [14], Proposition 1.14.

Proposition 5.4. A map $i: A \rightarrow B$ in $\operatorname{Sp}\left(\hat{\mathcal{S}}_{* G}\right)$ is a projective (trivial) cofibration if and only if $i_{0}: A_{0} \rightarrow B_{0}$ and the induced maps $j_{n}: A_{n} \amalg_{S^{1} \wedge A_{n-1}} S^{1} \wedge B_{n-1} \rightarrow B_{n}$ for $n \geqslant 1$ are (trivial) cofibrations in $\hat{\mathcal{S}}_{* G}$.

Proposition 5.5. The projective weak equivalences, projective fibrations and projective cofibrations define a left proper fibrantly generated simplicial model structure on $\operatorname{Sp}\left(\hat{\mathcal{S}}_{* G}\right)$. 
Proof. That we obtain a left proper fibrantly generated model structure can be proven in essentially the same way as Theorem 1.13 in [14]. In order to show the factorization axiom one uses a cosmall object argument and the fact that $\hat{\mathcal{S}}_{* G}$ is fibrantly generated. It remains to prove that this model structure is simplicial. We have defined tensor and cotensor objects for $\operatorname{Sp}\left(\hat{\mathcal{S}}_{* G}\right)$ above. Let $i: A \rightarrow B$ be a cofibration of simplicial sets and $p: X \rightarrow Y$ a projective fibration in $\operatorname{Sp}\left(\hat{\mathcal{S}}_{* G}\right)$. We have to show that the induced map

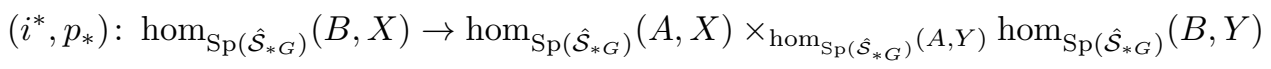

is a projective fibration in $\operatorname{Sp}\left(\hat{\mathcal{S}}_{* G}\right)$, which is trivial if either $i$ or $p$ is trivial. For $n \geqslant 0$, the $n$th map $\left(i^{*}, p_{*}\right)_{n}$ is given by the map of pointed profinite spaces

$$
\left(i^{*}, p_{n *}\right): \operatorname{hom}_{\hat{\mathcal{S}}_{* G}}\left(B, X_{n}\right) \rightarrow \operatorname{hom}_{\hat{\mathcal{S}}_{* G}}\left(A, X_{n}\right) \times_{\operatorname{hom}_{\hat{\mathcal{S}}_{* G}}\left(A, Y_{n}\right)} \operatorname{hom}_{\hat{\mathcal{S}}_{* G}}\left(B, Y_{n}\right) .
$$

The model structure on $\hat{\mathcal{S}}_{* G}$ is simplicial. This implies that $\left(i^{*}, p_{n *}\right)$ is a fibration, since $i$ is a cofibration and $p_{n}$ is a fibration. Moreover, $\left(i^{*}, p_{n *}\right)$ is a weak equivalence if either $i$ or $p_{n}$ is a weak equivalence. Since projective weak equivalences and projective fibrations are determined levelwise, this shows that $\left(i^{*}, p_{*}\right)$ is a projective fibration which is a trivial projective fibration if either $i$ or $p$ is trivial.

In the second step we enlarge the class of weak equivalences by localizing the projective model structure.

Definition 5.6. A profinite $G$-spectrum $E \in \operatorname{Sp}\left(\hat{\mathcal{S}}_{* G}\right)$ is called an $\Omega$-spectrum if each $E_{n}$ is fibrant in $\hat{\mathcal{S}}_{* G}$ and the adjoint structure maps

$$
E_{n} \rightarrow \Omega E_{n+1}=\operatorname{hom}_{\hat{\mathcal{S}}_{* G}}\left(S^{1}, E_{n+1}\right)
$$

are weak equivalences in $\hat{\mathcal{S}}_{* G}$ for all $n \geqslant 0$. A map $f: X \rightarrow Y$ of profinite $G$-spectra is called

- a (stable) equivalence if any projective cofibrant replacement $Q_{G} f: Q_{G} X \rightarrow$ $Q_{G} Y$ in $\operatorname{Sp}\left(\hat{\mathcal{S}}_{* G}\right)$ induces a weak equivalence of mapping spaces

$$
\operatorname{map}_{\mathrm{Sp}\left(\hat{\mathcal{S}}_{* G}\right)}\left(Q_{G} Y, E\right) \rightarrow \operatorname{map}_{\mathrm{Sp}\left(\hat{\mathcal{S}}_{* G}\right)}\left(Q_{G} X, E\right)
$$

for all $\Omega$-spectra $E$ in $\operatorname{Sp}\left(\hat{\mathcal{S}}_{* G}\right)$;

- a (stable) cofibration if and only if it is a projective cofibration;

- a (stable) fibration if it has the right lifting property with respect to all maps that are stable equivalences and stable cofibrations.

Theorem 5.7. The classes of stable equivalences, fibrations, and cofibrations of Definition 5.6 provide $\operatorname{Sp}\left(\hat{\mathcal{S}}_{* G}\right)$ with a stable simplicial model structure. The fibrant profinite $G$-spectra are exactly the $\Omega$-spectra in $\operatorname{Sp}\left(\hat{\mathcal{S}}_{* G}\right)$. Its underlying profinite spectra are $\Omega$-spectra in $\operatorname{Sp}\left(\hat{\mathcal{S}}_{*}\right)$. A map in $\operatorname{Sp}\left(\hat{\mathcal{S}}_{* G}\right)$ between $\Omega$-spectra is a stable equivalence if and only if it is a projective weak equivalence. We denote its homotopy category by $\hat{\mathcal{H}}_{G}$.

Proof. In order to show that there is a stable model structure on $\operatorname{Sp}\left(\hat{\mathcal{S}}_{* G}\right)$ we apply again the dual methods of Hovey [14]. We know from Theorem 3.4 that $\hat{\mathcal{S}}_{* G}$ satisfies 
the necessary properties. From Lemma 5.2 we know that smashing with $S^{1}$ is a left Quillen endofunctor on $\operatorname{Sp}\left(\hat{\mathcal{S}}_{* G}\right)$. By Proposition 5.5, we know that the projective model structure on $\operatorname{Sp}\left(\hat{\mathcal{S}}_{* G}\right)$ is simplicial. By localizing the projective model structure with respect to the $\Omega$-spectra in $\operatorname{Sp}\left(\hat{\mathcal{S}}_{* G}\right)$, we obtain a stable model structure on $\operatorname{Sp}\left(\hat{\mathcal{S}}_{* G}\right)$.

The $\Omega$-spectra are the fibrant objects in the stable model structure, since they are the local objects. Since weak equivalences and fibrations in $\hat{\mathcal{S}}_{* G}$ are determined by their underlying maps in $\hat{\mathcal{S}}_{*}$, this shows that the fibrant objects in $\operatorname{Sp}\left(\hat{\mathcal{S}}_{* G}\right)$ are exactly the profinite $G$-spectra whose underlying spectrum is fibrant in $\operatorname{Sp}\left(\hat{\mathcal{S}}_{*}\right)$.

The assertion on stable equivalences between $\Omega$-spectra in $\operatorname{Sp}\left(\hat{\mathcal{S}}_{* G}\right)$ follows from the general theory of Bousfield localizations.

Corollary 5.8. Let $K$ be a closed subgroup of the profinite group $G$. If $X$ is an $\Omega$-spectrum in $\operatorname{Sp}\left(\hat{\mathcal{S}}_{* G}\right)$, then its restriction to a profinite $K$-spectrum is also an $\Omega$ spectrum in $\operatorname{Sp}\left(\hat{\mathcal{S}}_{* K}\right)$.

Proof. Since weak equivalences and fibrations for $\hat{\mathcal{S}}_{* G}$ and $\hat{\mathcal{S}}_{* K}$ are determined by their underlying maps in $\hat{\mathcal{S}}_{*}$, the assertion follows from the definition of an $\Omega$ spectrum.

\section{Proposition 5.9.}

(a) The forgetful functor $\operatorname{Sp}\left(\hat{\mathcal{S}}_{* G}\right) \rightarrow \operatorname{Sp}\left(\hat{\mathcal{S}}_{*}\right)$ sends $\Omega$-spectra to $\Omega$-spectra and preserves stable equivalences between $\Omega$-spectra.

(b) The composition of forgetful functors $\operatorname{Sp}\left(\hat{\mathcal{S}}_{* G}\right) \rightarrow \operatorname{Sp}\left(\hat{\mathcal{S}}_{*}\right) \rightarrow \operatorname{Sp}\left(\mathcal{S}_{*}\right)$, which we also denote by $|\cdot|: \operatorname{Sp}\left(\hat{\mathcal{S}}_{* G}\right) \rightarrow \operatorname{Sp}\left(\mathcal{S}_{*}\right)$, sends $\Omega$-spectra to $\Omega$-spectra and preserves stable equivalences between $\Omega$-spectra.

Proof. (a) In the model structures on $\operatorname{Sp}\left(\hat{\mathcal{S}}_{* G}\right)$ and $\operatorname{Sp}\left(\hat{\mathcal{S}}_{*}\right)$, stable equivalences between $\Omega$-spectra are exactly the projective equivalences. By Theorem 5.7, the underlying profinite spectrum of an $\Omega$-spectrum in $\operatorname{Sp}\left(\hat{\mathcal{S}}_{* G}\right)$ is an $\Omega$-spectrum in $\operatorname{Sp}\left(\hat{\mathcal{S}}_{*}\right)$. Since projective equivalences are maps that are levelwise weak equivalences, the assertion follows from the fact that the forgetful functor $\hat{\mathcal{S}}_{* G} \rightarrow \hat{\mathcal{S}}_{*}$ preserves weak equivalences between fibrant objects.

(b) The second assertion follows from the first and from Proposition 4.7.

\subsection{Homotopy groups of fibrant profinite $G$-spectra}

Definition 5.10. Let $X$ be an $\Omega$-spectrum in $\operatorname{Sp}\left(\hat{\mathcal{S}}_{* G}\right)$. Its underlying profinite spectrum is fibrant in $\operatorname{Sp}\left(\hat{\mathcal{S}}_{*}\right)$, and, for $n \in \mathbb{Z}$, we define the $n$th homotopy group of $X$ to be the $n$th homotopy group of its underlying fibrant profinite spectrum.

Note that in Theorem 5.7 we did not show that the stable equivalences in $\operatorname{Sp}\left(\hat{\mathcal{S}}_{* G}\right)$ are determined by their underlying maps in $\operatorname{Sp}\left(\hat{\mathcal{S}}_{*}\right)$. If we start with an arbitrary profinite $G$-spectrum $Y$, we do not claim that the homotopy groups of the underlying profinite spectrum are isomorphic to the homotopy groups of $R_{G} Y$, where $R_{G}$ denotes a functorial fibrant replacement in $\operatorname{Sp}\left(\hat{\mathcal{S}}_{* G}\right)$. The point is that, since $Y$ is not fibrant, the fibrant replacement functors in $\operatorname{Sp}\left(\hat{\mathcal{S}}_{*}\right)$ and $\operatorname{Sp}\left(\hat{\mathcal{S}}_{* G}\right)$ may send it to profinite spectra which are not stably equivalent in $\operatorname{Sp}\left(\hat{\mathcal{S}}_{*}\right)$. 
But if we start with a fibrant profinite $G$-spectrum $X$, i.e., an $\Omega$-spectrum in $\operatorname{Sp}\left(\hat{\mathcal{S}}_{* G}\right)$, as in Definition 5.10 , then the homotopy groups of $X$ as a profinite $G$ spectrum are canonically isomorphic to the homotopy groups of its underlying $\Omega$ spectrum in $\operatorname{Sp}\left(\hat{\mathcal{S}}_{*}\right)$ by Proposition 5.9.

Moreover, the $G$-action on the $\Omega$-spectrum $X$ in $\operatorname{Sp}\left(\hat{\mathcal{S}}_{* G}\right)$ induces a $G$-action on each homotopy group of $X$.

Proposition 5.11. Let $X$ be an $\Omega$-spectrum in $\operatorname{Sp}\left(\hat{\mathcal{S}}_{* G}\right)$. Then each homotopy group $\pi_{n} X$ is a profinite $G$-module for every $n \in \mathbb{Z}$.

Proof. Since the underlying spectrum of $X$ is an $\Omega$-spectrum in $\operatorname{Sp}\left(\hat{\mathcal{S}}_{*}\right)$, Lemma 4.8 shows that $\pi_{n} X$ is isomorphic to $\pi_{n+k} X_{k}$ for any $k \geqslant 0$ such that $n+k \geqslant 0$. By Proposition 3.9, the groups $\pi_{n+k} X_{k}$ are profinite $G$-modules.

Remark 5.12. Let $X$ be again an $\Omega$-spectrum in $\operatorname{Sp}\left(\hat{\mathcal{S}}_{* G}\right)$. Let $Z$ be a spectrum in $\operatorname{Sp}\left(\mathcal{S}_{*}\right)$ which is isomorphic to a colimit of suspended sphere spectra $\mathbb{S}^{n_{\alpha}}$ for integers $n_{\alpha} \in \mathbb{Z}$ indexed by a filtered category. For such a spectrum $Z$, the generalized cohomology groups $X^{k} Z$ of (8) of $Z$ with coefficients in the underlying fibrant profinite spectrum of $X$, inherit a $G$-action from $X$. By Proposition 5.11, each

$$
\operatorname{Hom}_{\mathcal{S} \mathcal{H}}\left(\mathbb{S}^{n}, X\right)=\pi_{n} X
$$

is a profinite $G$-module. Hence (8) provides $X^{k} Z$ with the structure of a profinite $G$-module. Moreover, we may consider (9) as an isomorphism of profinite $G$-modules between $X^{k} Z$ and $|X|^{k} Z$.

\subsection{Homotopy limits of fibrant profinite $G$-spectra}

Let $I$ be a small category and let $X(-)$ be a functor from $I$ to the full subcategory of $\Omega$-spectra in $\operatorname{Sp}\left(\hat{\mathcal{S}}_{* G}\right)$. The homotopy inverse limit of the diagram $X(-)$ in $\operatorname{Sp}\left(\hat{\mathcal{S}}_{* G}\right)$ is again defined levelwise for each space. For each $n \geqslant 0$ and each $i \in I$, the pointed profinite $G$-space $X_{n}(i):=X(i)_{n}$ is fibrant. So for every $n \geqslant 0$, the homotopy limit $\operatorname{holim}_{i \in I} X_{n}(i)$ in $\hat{\mathcal{S}}_{* G}$ is a fibrant pointed profinite $G$-space by Lemma 3.11 and there is a natural isomorphism in $\hat{\mathcal{S}}_{* G}$

$$
\operatorname{holim}_{i \in I} \Omega\left(X_{n}(i)\right) \cong \Omega\left(\operatorname{holim}_{i \in I} X_{n}(i)\right) .
$$

Since each $X(i)$ is an $\Omega$-spectrum in $\operatorname{Sp}\left(\hat{\mathcal{S}}_{* G}\right)$ and since holim $i \in I$ preserves weak equivalences between fibrant objects by Lemma 3.11, for each $n \geqslant 0$, we obtain a weak equivalence in $\hat{\mathcal{S}}_{* G}$

$$
\operatorname{holim}_{i \in I} X_{n}(i) \stackrel{\sim}{\rightarrow} \operatorname{holim}_{i \in I} \Omega X_{n}(i) \cong \Omega \operatorname{holim}_{i \in I} X_{n}(i) .
$$

Hence together with these structure maps the sequence of fibrant pointed profinite $G$-spaces $\operatorname{holim}_{i \in I} X_{n}(i)$ defines a $\Omega$-spectrum in $\operatorname{Sp}\left(\hat{\mathcal{S}}_{* G}\right)$ that we denote by $\operatorname{holim}_{i \in I} X(i)$ and call the homotopy limit of the diagram $X(-)$.

Lemma 5.13. Let $f: X(-) \rightarrow Y(-)$ be a natural transformation of functors from a small category I to the full subcategory of $\Omega$-spectra in $\operatorname{Sp}\left(\hat{\mathcal{S}}_{* G}\right)$. If $f(i): X(i) \rightarrow Y(i)$ is an equivalence in $\operatorname{Sp}\left(\hat{\mathcal{S}}_{* G}\right)$ for every $i \in I$, then the induced map

$$
\operatorname{holim}_{i \in I} X(i) \rightarrow \operatorname{holim}_{i \in I} Y(i)
$$

is an equivalence of $\Omega$-spectra in $\operatorname{Sp}\left(\hat{\mathcal{S}}_{* G}\right)$. 
Proof. We already know that holim sends a small diagram of $\Omega$-spectra in $\operatorname{Sp}\left(\hat{\mathcal{S}}_{* G}\right)$ to an $\Omega$-spectrum in $\operatorname{Sp}\left(\hat{\mathcal{S}}_{* G}\right)$. Since holim is constructed termwise and since stable equivalences between $\Omega$-spectra are exactly the projective equivalences in $\operatorname{Sp}\left(\hat{\mathcal{S}}_{* G}\right)$, the assertion follows from the corresponding result for $\hat{\mathcal{S}}_{* G}$ given in Lemma 3.11.

Lemma 5.14. Let $X(-): I \rightarrow \operatorname{Sp}\left(\hat{\mathcal{S}}_{* G}\right)$ be a small cofiltering diagram of $\Omega$-spectra in $\operatorname{Sp}\left(\hat{\mathcal{S}}_{* G}\right)$. Then the isomorphism of Lemma 4.10

$$
\pi_{q}\left(\left|\operatorname{holim}_{i \in I} X(i)\right|\right) \cong \lim _{i \in I} \pi_{q}(|X(i)|)
$$

is an isomorphism of profinite $G$-modules for every $q \in \mathbb{Z}$.

Proof. Since the $\Omega$-spectrum holim $\operatorname{lif}_{i \in I} X(i)$ in $\operatorname{Sp}\left(\hat{\mathcal{S}}_{* G}\right)$ is constructed levelwise, the assertion follows from Lemma 3.12.

\section{4. $\quad G$-spectra with finite homotopy groups}

Theorem 5.15. Let $G$ be a strongly complete profinite group. Let $X \in \operatorname{Sp}\left(\mathcal{S}_{*}\right)$ be a spectrum such that each space $X_{n}$ is a pointed $G$-space and the $G$-actions are compatible with the bonding maps. We assume that the homotopy groups of $X$ are all finite groups. Then there is a G-equivariant map

$$
\varphi^{s}: X \rightarrow F_{G}^{s} X
$$

of spectra from $X$ to a profinite $G$-spectrum $F_{G}^{s} X$ built of simplicial finite discrete $G$ sets such that $F_{G}^{s} X$ is fibrant in $\operatorname{Sp}\left(\hat{\mathcal{S}}_{* G}\right)$ and $\varphi^{s}$ is a stable equivalence of underlying spectra. In particular, $\varphi^{s}$ induces an isomorphism $\pi_{*} X \cong \pi_{*}\left|F_{G}^{s} X\right|$ of the homotopy groups of underlying spectra.

The assignment $X \mapsto F_{G}^{s} X$ is functorial in the sense that given a $G$-equivariant map $h: X \rightarrow Y$ between $G$-spectra whose spaces are pointed $G$-spaces and whose homotopy groups are finite, there is a map $F_{G}^{s}(h)$ in $\operatorname{Sp}\left(\hat{\mathcal{S}}_{* G}\right)$ such that the following diagram of underlying spectra commutes

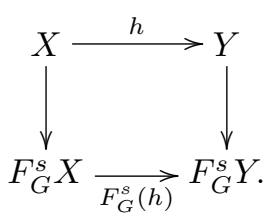

Proof. The proof is similar to the one of Theorem 4.11 using the replacement functor of Lemma 3.13. We can assume that $X$ is an $\Omega$-spectrum in $\operatorname{Sp}\left(\mathcal{S}_{*}\right)$. Since the homotopy groups of $X$ are finite, each of the groups $\pi_{k}\left(X_{n}\right)$ is a finite group for all $k, n \geqslant 0$. Moreover, since $X$ is a spectrum, we can assume that each $X_{n}$ is a simple connected pointed $G$-space. Hence we can apply the functor $F_{G}$ of Lemma 3.13 to each space $X_{n}$ and obtain the profinite spectrum $F_{G}^{s} X$. By Lemma 3.14, the induced maps

$$
F_{G} X_{n} \rightarrow \Omega\left(F_{G} X_{n+1}\right)
$$

are weak equivalences in $\hat{\mathcal{S}}_{* G}$. Since each $F_{G} X_{n}$ is fibrant in $\hat{\mathcal{S}}_{* G}$, this implies that the profinite $G$-spectrum $F_{G}^{s} X$ is an $\Omega$-spectrum in $\operatorname{Sp}\left(\hat{\mathcal{S}}_{*}\right)$. The $i$ th stable homotopy 
group of the $\Omega$-spectrum $F_{G}^{s} X$ is given by any of the isomorphic finite groups

$$
\pi_{i+n}\left(F_{G} X_{n}\right) \cong \pi_{i+n}\left(X_{n}\right)
$$

for positive $n$ such that $i+n \geqslant 0$. This shows that $X \rightarrow F_{G}^{s} X$ induces an isomorphism $\pi_{*} X \cong \pi_{*}\left|F_{G}^{s} X\right|$. Finally, the functoriality of $F_{G}^{s}$ follows from the functoriality of $F_{G}$.

\section{References}

[1] M. Artin, B. Mazur, Etale homotopy, Lecture Notes in Mathematics 100, Springer, Berlin-New York, 1969.

[2] A. K. Bousfield, E. M. Friedlander, Homotopy of $\Gamma$-spaces, spectra and bisimplicial sets, in: Geometric Applications of Homotopy Theory (Evanston, IL, 1977), vol. II, Lecture Notes in Mathematics 658, Springer, Berlin, 1978, 80130.

[3] A. K. Bousfield, D. M. Kan, Homotopy limits, Completions and Localizations, Lecture Notes in Mathematics 304, Springer-Verlag, Berlin-New York, 1972.

[4] J. D. Christensen, D. C. Isaksen, Duality and pro-spectra, Alg. Geom. Topol. 4 (2004), 781-812.

[5] D. G. Davis, Homotopy fixed points for $L_{K(n)}\left(E_{n} \wedge X\right)$ using the continuous action, J. Pure Appl. Algebra 206 (2006), 322-354.

[6] F.-X. Dehon, J. Lannes, Sur les espaces fonctionnels dont la source est le classifiant d'un groupe de Lie compact commutatif, Inst. Hautes Études Sci. Publ. Math. 89 (1999), 127-177.

[7] F.-X. Dehon, Cobordisme complexe des espaces profinis et foncteur $T$ de Lannes, Mém. Soc. Math. Fr. 98 (2004), vi+198 pp.

[8] E. S. Devinatz, M. J. Hopkins, Homotopy fixed point spectra for closed subgroups of the Morava stabilizer groups, Topology 43 (2004), no. 1, 1-47.

[9] W. G. Dwyer, D. M. Kan, Homotopy theory and simplicial groupoids, Nederl. Akad. Wetensch. Indag. Math. 46 (1984), 379-385.

[10] P. G. Goerss, Homotopy Fixed Points for Galois Groups, in: The Cech centennial (Boston, 1993), Contemp. Math. 181, 1995, 187-224.

[11] P. G. Goerss, J. F. Jardine, Simplicial Homotopy Theory, Birkhäuser Verlag, Basel, 1999.

[12] P. S. Hirschhorn, Model Categories and Their Localizations, Mathematical Surveys and Monographs 99, Amer. Math. Soc., Providence, 2003.

[13] M. Hovey, Model Categories, Mathematical Surveys and Monographs 63, Amer. Math. Soc., 1999.

[14] M. Hovey, Spectra and symmetric spectra in general model categories, J. Pure Appl. Algebra 165 (2001), 63-127.

[15] M. Hovey, B. Shipley, J. Smith, Symmetric spectra, J. Amer. Math. Soc. 13 (2000), 149-208.

[16] F. Morel, Ensembles profinis simpliciaux et interprétation géométrique du foncteur T, Bull. Soc. Math. France 124 (1996), 347-373. 
[17] N. Nikolov, D. Segal, On finitely generated profinite groups. I. Strong completeness and uniform bounds, Ann. of Math. 165 (2007), 171-238.

[18] G. Quick, Stable étale realization and étale cobordism, Adv. Math. 214 (2007), 730-760.

[19] G. Quick, Profinite homotopy theory, Doc. Math. 13 (2008), 585-612.

[20] G. Quick, Continuous group actions on profinite spaces, J. Pure Appl. Algebra 215 (2011), 1024-1039.

[21] G. Quick, Some remarks on profinite completion of spaces, to appear in: H. Nakamura, F. Pop, L. Schneps, A. Tamagawa (eds.), Galois-Teichmueller theory and Arithmetic Geometry (Kyoto 2010), Adv. Stud. Pure Math. 63, Math. Soc. Japan., Tokyo, 2012, 413-448.

[22] G. Quick, Continuous homotopy fixed points for Lubin-Tate spectra, Homology Homotopy Appl. 15(1) (2013), 191-222.

[23] D. G. Quillen, Homotopical algebra, Lecture Notes in Mathematics 43, Springer-Verlag, Berlin-New York, 1967.

[24] D. Quillen, An application of simplicial profinite groups, Comment. Math. Helv. 44 (1969), 45-60.

[25] L. Ribes, P. Zalesskii, Profinite Groups, Ergebnisse der Mathematik und ihrer Grenzgebiete 40, Springer-Verlag, Berlin, 2000.

[26] J. P. Serre, Cohomologie Galoisienne, Lecture Notes in Mathematics 5, Springer-Verlag, Berlin-New York, 1965.

[27] D. Sullivan, Genetics of Homotopy Theory and the Adams Conjecture, Ann. of Math. 100 (1974), 1-79.

[28] R. W. Thomason, Algebraic K-Theory and Étale Cohomology, Ann. Sci. E.N.S. 18 (1985), 437-552.

Gereon Quick gquick@math.harvard.edu

Department of Mathematics, Harvard University, One Oxford Street, Cambridge, MA 02138, USA 